\title{
Subexponential decay and regularity estimates for eigenfunctions of localization operators
}

\author{
Federico Bastianoni ${ }^{1}$. Nenad Teofanov ${ }^{2}$ \\ Received: 2 May 2020 / Revised: 23 September 2020 / Accepted: 28 September 2020 / \\ Published online: 17 February 2021 \\ (c) The Author(s) 2021
}

\begin{abstract}
We consider time-frequency localization operators $A_{a}^{\varphi_{1}, \varphi_{2}}$ with symbols $a$ in the wide weighted modulation space $M_{w}^{\infty}\left(\mathbb{R}^{2 d}\right)$, and windows $\varphi_{1}, \varphi_{2}$ in the Gelfand-Shilov space $\mathcal{S}^{(1)}\left(\mathbb{R}^{d}\right)$. If the weights under consideration are of ultra-rapid growth, we prove that the eigenfunctions of $A_{a}^{\varphi_{1}, \varphi_{2}}$ have appropriate subexponential decay in phase space, i.e. that they belong to the Gelfand-Shilov space $\mathcal{S}^{(\gamma)}(\mathbb{R})$, where the parameter $\gamma \geq 1$ is related to the growth of the considered weight. An important role is played by $\tau$-pseudodifferential operators $O p_{\tau}(\sigma)$. In that direction we show convenient continuity properties of $O p_{\tau}(\sigma)$ when acting on weighted modulation spaces. Furthermore, we prove subexponential decay and regularity properties of the eigenfunctions of $O p_{\tau}(\sigma)$ when the symbol $\sigma$ belongs to a modulation space with appropriately chosen weight functions. As an auxiliary result we also prove new convolution relations for (quasi-)Banach weighted modulation spaces.
\end{abstract}

Keywords Time-frequency analysis · Pseudodifferential operators · Schatten classes $\cdot$ Modulation spaces $\cdot$ Gelfand-Shilov spaces

Mathematics Subject Classification 47G30 - 47B10 $\cdot 46 \mathrm{~F} 05 \cdot 35 \mathrm{~S} 05$

\section{Introduction}

Localization operators can be considered as appropriate mathematical instrument for studying the signals with prescribed characteristics in a given region of the time-

Federico Bastianoni

federico.bastianoni@polito.it

Nenad Teofanov

nenad.teofanov@dmi.uns.ac.rs

1 Dipartimento di Scienze Matematiche, Politecnico di Torino, Corso Duca degli Abruzzi 24, 10129, Torino, Italy

2 Department of Mathematics and Informatics, University of Novi Sad, Novi Sad, Serbia

Birkhäuser 
frequency plane [36]. They are defined as integral transforms determined by a suitable choice of a symbol $a$ and window functions $\varphi_{1}, \varphi_{2}$. In this paper we examine the conditions which the symbol and windows of an operator should satisfy to ensure an ultra-rapid decay and regularity of its eigenfunctions. It is known that the eigenfunctions of time-frequency localization operators, also known as Daubechies operators and denoted by $A_{a}^{\varphi_{1}, \varphi_{2}}$, with Gaussian windows

$$
\varphi_{1}(t)=\varphi_{2}(t)=\pi^{-d / 4} \exp \left(-t^{2} / 2\right) \text { and with a radial symbol } a \in L^{1}\left(\mathbb{R}^{2 d}\right),
$$

are Hermite functions, i.e. they have superexponential decay in phase space [16]. In a different terminology, those eigenfunctions belong to the smallest projective GelfandShilov space $\mathcal{S}^{\{1 / 2\}}(\mathbb{R})$ (cf. Definition 2.7).

The investigations in $[16,36]$ are motivated by some questions in signal analysis. However, localization operators appear in different mathematical contexts. For example, they were used (under the name anti-Wick operators) in the study of quantization problem in quantum mechanics, cf. [3,37]. In abstract harmonic analysis, localization operators on a locally compact group $G$ and Lebesgue spaces $L^{p}(G), 1 \leq p \leq \infty$, were studied in [50]. We also mention their presence in the form of Toeplitz operators in complex analysis [4]. Here we do not intend to discuss different manifestations of localization operators and refer to e.g. [17] for a survey.

Our results fits in the framework of time-frequency analysis. An important step forward in that direction was made by the seminal paper [9]. Thereafter the subject has been considered by many authors including $[1,5,10,13,26,41,44]$, where among others one can find different continuity, Schatten class and lifting properties of localization operators. The time-frequency analysis approach is based on the use of modulation spaces as appropriate functional analytic framework. Another issue established in [5,9] is the identification of localization operators as Weyl pseudodifferential operators.

The focus of this paper is to consider the properties of eigenfunctions of compact localization operators. Our investigations are inspired by the recent work [2]. Indeed, there it is shown that if the symbol $a$ belongs to the modulation space $M_{v_{s} \otimes 1}^{\infty}\left(\mathbb{R}^{2 d}\right)$, $s>0$ (see Definition 2.17) and $\varphi_{1}, \varphi_{2} \in \mathcal{S}\left(\mathbb{R}^{d}\right)$, then the eigenfunctions of $A_{a}^{\varphi_{1}, \varphi_{2}}$ are actually Schwartz functions. Moreover, similar result is proved for the Weyl pseudodifferential operators whose symbol belongs to $M_{v_{s} \otimes v_{t}}^{\infty, 1}\left(\mathbb{R}^{2 d}\right)$, for some $s>0$ and every $t>0$, cf. [2, Proposition 3.6]. Here $v_{s}(z)=\left(1+|z|^{2}\right)^{s / 2}, s \in \mathbb{R}, z \in \mathbb{R}^{d}$.

We extend the framework of the Schwartz space of test functions and its dual space of tempered distributions given in [2] by replacing it with a more subtle family of Gelfand-Shilov spaces and their duals, spaces of ultra-distributions. This is motivated by the fact that Gelfand-Shilov spaces describe smoothness and decay properties which go beyond the scope of the Schwartz space, cf. [7,22,23,34,35,40,44]. To that end, as an important technical tool, we consider a class of weights which contains the weights of subexponential growth, apart from polynomial type weights. As explained in [27], replacing polynomial weights with weights of faster growth at infinity is not a mere routine. Indeed, weights of ultra-rapid growth and decay give rise to ultradistributional framework for modulation spaces. To underline this difference, we sometimes refer to ultra-modulation spaces when modulation spaces are allowed to contain such ultra-distributions. 
One of the main tools in our analysis is the (cross-) $\tau$-Wigner distribution $W_{\tau}(f, g)$, $f, g \in L^{2}\left(\mathbb{R}^{d}\right)$, see Definition 2.11. The relation between $W_{\tau}(f, g)$ and the short-time Fourier transform $V_{g}(f)$ (another relevant time-frequency representation) serves as a bridge between properties of modulation spaces and $\tau$-pseudodifferential operators. More precisely, we extend the recent result [2, Theorem 3.3] to a more general class of operators and weights (Theorem 3.3). Although this result follows from [47, Theorem 3.1] and [45, Theorem A.2], our proof is independent and based on different arguments.

Our first main result concerns decay properties of the eigenfunctions of $\tau$ pseudodifferential operators. In fact, by using iterated actions of the operator we conclude that its eigenfunctions belong to the Gelfand-Shilov space $\mathcal{S}^{(\gamma)}\left(\mathbb{R}^{d}\right)$ (Theorem 3.7). As already mentioned, this gives an information about regularity and decay properties of eigenfunctions which can not be captured within the Schwartz class.

Finally, we use Theorem 3.7 and convolution relation for modulation spaces (Proposition 2.24) to show that the eigenfunctions of localization operators $A_{a}^{\varphi_{1}, \varphi_{2}}$ have appropriate subexponential decay in phase space if $a \in M_{w}^{\infty}\left(\mathbb{R}^{2 d}\right), \varphi_{1}, \varphi_{2} \in \mathcal{S}^{(1)}\left(\mathbb{R}^{d}\right)$, and if $w$ is of a certain ultra-rapid growth. We use the representation of localization operators as pseudodifferential operators. Evidently, the Weyl form of localization operators suggests to introduce and consider $\tau$-localization operators by using $\tau$ pseudodifferential operators and the (cross-) $\tau$-Wigner distribution. However, it turns out the such approach does not extend the class of localization operators given by Definition 2.15 (cf. Proposition 2.16).

We end this introduction with a brief report of the content of the paper. In Preliminaries we collect relevant background material. Apart from the review of known results, it contains some new results and proofs. In Sect. 3 we prove our main results: continuity properties of $\tau$-pseudodifferential operators on modulation spaces, estimates for eigenfunctions of $\tau$-pseudodifferential operators and decay and smoothness properties of eigenfunctions of localization operators. Appendix contains the proofs of two auxiliary technical results.

\subsection{Notation}

We denote the Euclidean scalar product on $\mathbb{R}^{d}$ by $x y:=x \cdot y$ and the Euclidean norm by $|x|:=\sqrt{x \cdot x}$. We put $\mathbb{N}_{0}:=\mathbb{N} \cup\{0\} . A \lesssim B$ means that for given constants $A$ and $B$ there exists a constant $c>0$ independent of $A$ and $B$ such that $A \leq c B$, and we write $A \asymp B$ if both $A \lesssim B$ and $B \lesssim A$.

We define the involution $g^{*}$ of a function $g$ by $g^{*}(t):=\overline{g(-t)}$. Given a function $f$ on $\mathbb{R}^{d}$ its Fourier transform is normalized to be

$$
\mathcal{F} f(\omega)=\hat{f}(\omega):=\int_{\mathbb{R}^{d}} e^{-2 \pi i x \omega} f(x) d x, \quad \omega \in \mathbb{R}^{d}
$$

Given two spaces $A$ and $B$, we denote by $A \hookrightarrow B$ the continuous embedding of $A$ into $B . \mathcal{S}\left(\mathbb{R}^{d}\right)$ denotes the Schwartz class, and its topological dual, the space of tempered distributions, is indicated by $\mathcal{S}^{\prime}\left(\mathbb{R}^{d}\right)$. By the brackets $\langle f, g\rangle$ we mean the extension of the $L^{2}$-inner product $\langle f, g\rangle:=\int f(t) \overline{g(t)} d t$ to any dual pair. 
Consider $0<p<\infty$ and a positive and measurable function $m$ on $\mathbb{R}^{d}$, then $L_{m}^{p}\left(\mathbb{R}^{d}\right)$ denotes the (quasi-)Banach space of measurable functions $f: \mathbb{R}^{d} \rightarrow \mathbb{C}$ such that

$$
\|f\|_{L_{m}^{p}}:=\left(\int_{\mathbb{R}^{d}}|f(x)|^{p} m(x)^{p} d x\right)^{1 / p}<+\infty
$$

modulus the equivalence relation $f \sim g \Leftrightarrow f(x)=g(x)$ for a.e. $x$. When $p=\infty$, $f \in L_{m}^{\infty}\left(\mathbb{R}^{d}\right)$ if $\|f\|_{L_{m}^{\infty}}:=$ ess sup $x_{x \in \mathbb{R}^{d}}|f(x)| m(x)<+\infty$, up to the equivalence relation defined above. If $m \equiv 1$, we use abbreviated notation $L^{p}\left(\mathbb{R}^{d}\right)=L_{1}^{p}\left(\mathbb{R}^{d}\right)$. If the restriction of $f$ to any compact set belongs to $L^{p}\left(\mathbb{R}^{d}\right)$, then we write $f \in L_{l o c}^{p}\left(\mathbb{R}^{d}\right)$.

For given Hilbert space $H$ and compact operator $T$ on $H$ its singular values $\left\{s_{k}(T)\right\}_{k=1}^{\infty}$ are the eigenvalues of $\left(T^{*} T\right)^{1 / 2}$, which is a positive and self-adjoint operator. The Schatten class $S_{p}(H)$, with $0<p<\infty$, is the set of all compact operators on $H$ such that their singular values are in $\ell^{p}$. For consistency, we define $S_{\infty}(H):=B(H)$, the set of all linear and bounded operators on $H$. We shall deal with $H=L^{2}\left(\mathbb{R}^{d}\right)$.

By $\sigma_{P}(T)$ we denote the point spectrum of the operator $T$. If $T$ is a compact mapping on $L^{2}\left(\mathbb{R}^{d}\right)$, then the spectral theory for compact operators yields $\sigma(T) \backslash\{0\}=\sigma_{P}(T) \backslash\{0\}$, where $\sigma(T)$ is the spectrum of the operator. For compact operators on $L^{2}\left(\mathbb{R}^{d}\right)$, we have $0 \in \sigma(T)$ and the point spectrum $\sigma_{P}(T) \backslash\{0\}$ (possibly empty) is at most a countable set.

A function $f \in L^{2}\left(\mathbb{R}^{d}\right) \backslash\{0\}$ is an eigenfunction of the operator $T$ if there exists $\lambda \in \mathbb{C}$ such that $T f=\lambda f$. We are interested in the properties of eigenfunctions of $A_{a}^{\varphi_{1}, \varphi_{2}}$ related to eigenvalues $\lambda \in \sigma_{P}\left(A_{a}^{\varphi_{1}, \varphi_{2}}\right) \backslash\{0\}$, whenever $\sigma_{P}\left(A_{a}^{\varphi_{1}, \varphi_{2}}\right) \backslash\{0\} \neq \varnothing$.

\section{Preliminaries}

In this section we collect background material and prove some auxiliary results. More precisely, we provide definitions and basic facts on weights, sequence spaces, Gelfand-Shilov spaces, time-frequency representations, pseudodifferential and localization operators, modulation spaces and Gabor frames. We also give some new results and proofs (Lemma 2.4, Proposition 2.16, Proposition 2.24) which will be used in Sect. 3.

\subsection{Weight functions}

By weight $m$ on $\mathbb{R}^{d}$ (or on $\mathbb{Z}^{d}$ ) we mean a positive function $m>0$ such that $m \in$ $L_{\text {loc }}^{\infty}\left(\mathbb{R}^{d}\right)$ and $1 / m \in L_{\text {loc }}^{\infty}\left(\mathbb{R}^{d}\right)$. A weight $m$ is said to be submultiplicative if it is even and

$$
m(x+y) \leq m(x) m(y), \quad \forall x \in \mathbb{R}^{d} .
$$

Given a weight $m$ on $\mathbb{R}^{d}$ and a positive function $v \in L_{l o c}^{\infty}\left(\mathbb{R}^{d}\right)$, we say that $m$ is $v$-moderate if

$$
m(x+y) \lesssim v(x) m(y), \quad \forall x, y \in \mathbb{R}^{d} .
$$


Therefore submultiplicative weights are moderate and the previous inequality implies the following estimates:

$$
v(-x)^{-1} \lesssim m(x) \lesssim v(x), \quad \forall x \in \mathbb{R}^{d}
$$

For a submultiplicative weight $v$ there are convenient ways to find a smooth weight $v_{0}$ which is equivalent to $v$ in the sense that there is a constant $C>0$ such that

$$
C^{-1} v_{0} \leq v \leq C v_{0}
$$

see e.g. $[15,25,44]$.

Next we introduce some weights which will be used in the sequel. Given $k, \gamma>0$ we define

$$
w_{k}^{\gamma}(x):=e^{k|x|^{1 / \gamma}}, \quad x \in \mathbb{R}^{d} .
$$

Sometimes we shall use the above expression for $k=0$ also, with obvious meaning. If $\gamma>1$ the above functions are called subexponential weights, and when $\gamma=1$ we write $w_{k}$ instead of $w_{k}^{1}$. Note that (sub-)exponential weights $w_{k}^{\gamma}$ are submultiplicative [this follows from (29)]. When $0<\gamma<1$ we obtain weights of super-exponential growth at infinity. We shall work with the following weight classes defined for $\gamma>0$ :

$$
\begin{aligned}
\mathscr{P}_{E}\left(\mathbb{R}^{d}\right) & :=\left\{m \text { weight on } \mathbb{R}^{d} \mid m \text { is } v-\text { moderate for some submultiplicative } v\right\}, \\
\mathscr{P}_{E, \gamma}\left(\mathbb{R}^{d}\right) & :=\left\{m \text { weight on } \mathbb{R}^{d} \mid m \text { is } w_{k}^{\gamma} \text { - moderate for some } k>0\right\}, \\
\mathscr{P}_{E, \gamma}^{0}\left(\mathbb{R}^{d}\right) & :=\left\{m \text { weight on } \mathbb{R}^{d} \mid m \text { is } w_{k}^{\gamma} \text { - moderate for every } k>0\right\} .
\end{aligned}
$$

For $0<\gamma_{2}<\gamma_{1}$ we have

$$
\mathscr{P}_{E, \gamma_{1}}^{0} \subseteq \mathscr{P}_{E, \gamma_{1}} \subseteq \mathscr{P}_{E, \gamma_{2}}^{0} \subseteq \mathscr{P}_{E}
$$

Moreover, for $0<\gamma<1$ we have $\mathscr{P}_{E}=\mathscr{P}_{E, \gamma}=\mathscr{P}_{E, \gamma}^{0}$; see [6, Remark 2.6] and [49]. The next lemma states that if $m \in \mathscr{P}_{E}$, then it is $w_{k}$-moderate fore some $k>0$ large enough. This implies $\mathscr{P}_{E}=\mathscr{P}_{E, 1}$.

Lemma 2.1 Let $m \in \mathscr{P}_{E}$. Then $m$ is $w_{k}$-moderate fore some $k>0$.

Proof The lemma is folklore, and we refer e.g. to Gröchenig [25], Cappiello and Toft [6], and Toft $[47,48]$ for its proof.

We remark that $\mathscr{P}_{E}$ contains the weights of polynomial type, i.e. weights moderate with respect to some polynomial.

In the sequel $\mathscr{P}_{E, \gamma}^{*}$ means $\mathscr{P}_{E, \gamma}$ or $\mathscr{P}_{E, \gamma}^{0}$. The following lemma follows by easy calculations and we leave the proof for the reader (see also [44]). Observe that due to the equality $\mathscr{P}_{E, 1}=\mathscr{P}_{E, \gamma}=\mathscr{P}_{E, \gamma}^{0}, 0<\gamma<1$, it is sufficient to consider $\gamma \geq 1$.

Lemma 2.2 Consider $\gamma>0$. Then $\mathscr{P}_{E, \gamma}^{*}\left(\mathbb{R}^{d}\right)$ is a group under the pointwise multiplication and with the identity $m \equiv 1$. 
Given a function $f$ defined on $\mathbb{R}^{2 d}$ we denote its restrictions to $\mathbb{R}^{d} \times\{0\}$ and $\{0\} \times \mathbb{R}^{d}$ as follows:

$$
\left.f\right|_{1}(x):=f(x, 0),\left.\quad f\right|_{2}(\omega):=f(0, \omega), \quad x, \omega \in \mathbb{R}^{d} .
$$

Given two functions $g, h$ defined on $\mathbb{R}^{d}$ their tensor product is the function on $\mathbb{R}^{2 d}$ defined in the following manner:

$$
g \otimes h(x, \omega):=g(x) h(\omega), \quad(x, \omega) \in \mathbb{R}^{2 d} .
$$

The families $\mathscr{P}_{E, \gamma}^{*}$ turn out to be closed under restrictions and tensor products in the sense of the following lemma. The proof is omitted, since it follows from definitions and properties of the Euclidean norm.

Lemma 2.3 Consider $\gamma>0$ :

(i) if $m \in \mathscr{P}_{E, \gamma}^{*}\left(\mathbb{R}^{2 d}\right)$, then $\left.m\right|_{1},\left.m\right|_{2} \in \mathscr{P}_{E, \gamma}^{*}\left(\mathbb{R}^{d}\right)$;

(ii) if $m, w \in \mathscr{P}_{E, \gamma}^{*}\left(\mathbb{R}^{d}\right)$, then $m \otimes w \in \mathscr{P}_{E, \gamma}^{*}\left(\mathbb{R}^{2 d}\right)$.

Next we exhibit a lemma which will play a key role in the sequel, see Proposition 3.7. The proof is given in the appendix.

Lemma 2.4 Consider $\gamma \geq 1, r, s \geq 0, \tau \in[0,1]$ and

$$
t \geq \begin{cases}r+s \tau^{1 / \gamma} & \text { if } 1 / 2 \leq \tau \leq 1 \\ r+s\left(1+\tau^{2}\right)^{1 / 2 \gamma} & \text { if } 0 \leq \tau<1 / 2\end{cases}
$$

Then for every $x, \omega, y, \eta \in \mathbb{R}^{d}$ the following estimate holds true:

$$
\frac{w_{r+s}^{\gamma}(x, \omega)}{w_{r}^{\gamma}(y, \eta)} \leq w_{s}^{\gamma} \otimes w_{t}^{\gamma}(((1-\tau) x+\tau y, \tau \omega+(1-\tau) \eta),(\omega-\eta, y-x)) \text {. }
$$

We finish this subsection by introducing some polynomial weights which will be used in Theorem 3.4 and Lemma 3.5. Let $\tau \in[0,1]$ and $u \geq 0$, then we define the weights of polynomial type

$$
\begin{aligned}
& v_{u}(x, \omega):=\langle(x, \omega)\rangle^{u}=\left(1+|(x, \omega)|^{2}\right)^{u / 2}, \quad(x, \omega) \in \mathbb{R}^{2 d}, \\
& m_{u}^{\tau}((x, \omega),(y, \eta)):=(1+|x-\tau \eta|+|\omega+(1-\tau) y|)^{u}, \quad(x, \omega),(y, \eta) \in \mathbb{R}^{2 d} .
\end{aligned}
$$

Remark 2.5 If $v_{u}$ and $m_{u}^{\tau}$ are given by (4) and (5) respectively, then we notice that

$$
m_{u}^{\tau}((x, \omega),(y, \eta)) \lesssim v_{u} \otimes v_{u}((x, \omega),(y, \eta)), \quad \forall(x, \omega),(y, \eta) \in \mathbb{R}^{2 d} .
$$

which will be used in Lemma 3.5. Indeed:

$m_{u}^{\tau}((x, \omega),(y, \eta))=(1+|x-\tau \eta|+|\omega+(1-\tau) y|)^{u}$ 


$$
\begin{aligned}
& \lesssim\left(1+(|x|+|\tau \eta|)^{2}+(|\omega|+|(1-\tau) y|)^{2}\right)^{u / 2} \\
& \lesssim\left(1+|x|^{2}+\tau^{2}|\eta|^{2}+|\omega|^{2}+(1-\tau)^{2}|y|^{2}\right)^{u / 2} \\
& \lesssim\left(1+|(x, \omega)|^{2}+|(y, \eta)|^{2}\right)^{u / 2} \\
& \leq\left(1+|(x, \omega)|^{2}+|(y, \eta)|^{2}+|(x, \omega)|^{2}|(y, \eta)|^{2}\right)^{u / 2} \\
& =\left(1+|(x, \omega)|^{2}\right)^{u / 2}\left(1+|(y, \eta)|^{2}\right)^{u / 2}=v_{u} \otimes v_{u}((x, \omega),(y, \eta)) .
\end{aligned}
$$

\subsection{Spaces of sequences}

Given $0<p, q \leq \infty$ and $m \in \mathscr{P}_{E}\left(\mathbb{Z}^{2 d}\right), \ell_{m}^{p, q}\left(\mathbb{Z}^{2 d}\right)$ is the set of all sequences $a=\left(a_{k, n}\right)_{k, n \in \mathbb{Z}^{d}}$ such that the (quasi-)norm

$$
\|a\|_{\ell_{m}^{p, q}}:=\left(\sum_{n \in \mathbb{Z}^{d}}\left(\sum_{k \in \mathbb{Z}^{d}}\left|a_{k, n}\right|^{p} m(k, n)^{p}\right)^{\frac{q}{p}}\right)^{\frac{1}{q}}
$$

(with obvious changes for $p=\infty$ or $q=\infty$ ) is finite.

When $p=q$ we recover the standard spaces of sequences $\ell_{m}^{p, p}\left(\mathbb{Z}^{2 d}\right)=\ell_{m}^{p}\left(\mathbb{Z}^{2 d}\right)$.

In the following proposition we collect some properties that we shall use later on, see $[20,21]$.

Proposition 2.6 (i) Inclusion relations: consider $0<p_{1} \leq p_{2} \leq \infty$ and let $m$ be any positive weight function on $\mathbb{Z}^{d}$. Then

$$
\ell_{m}^{p_{1}}\left(\mathbb{Z}^{d}\right) \hookrightarrow \ell_{m}^{p_{2}}\left(\mathbb{Z}^{d}\right) .
$$

(ii) Young's convolution inequality: consider $m, v \in \mathscr{P}_{E}\left(\mathbb{Z}^{d}\right)$ such that $v$ is submultiplicative and $m$ is $v$-moderate, $0<p, q, r \leq \infty$ with

$$
\frac{1}{p}+\frac{1}{q}=1+\frac{1}{r}, \quad \text { for } 1 \leq r \leq \infty
$$

and

$$
p=q=r, \text { for } 0<r<1 .
$$

Then for all $a \in \ell_{m}^{p}\left(\mathbb{Z}^{d}\right), b \in \ell_{v}^{q}\left(\mathbb{Z}^{d}\right)$, we have $a * b \in \ell_{m}^{r}\left(\mathbb{Z}^{d}\right)$, with

$$
\|a * b\|_{\ell_{m}^{r}} \leq C\|a\|_{\ell_{m}^{p}}\|b\|_{\ell_{v}^{q}}
$$

where the constant $C>0$ is independent of $p, q, r, a$ and $b$. If $m \equiv v \equiv 1$, then $C=1$.

(iii) Hölder's inequality: let $m$ be any positive weight function on $\mathbb{Z}^{d}$ and $0<$ $p, q, r \leq \infty$ such that $1 / p+1 / q=1 / r$. Then

$$
\ell_{m}^{p}\left(\mathbb{Z}^{d}\right) \cdot \ell_{1 / m}^{q}\left(\mathbb{Z}^{d}\right) \hookrightarrow \ell^{r}\left(\mathbb{Z}^{d}\right) .
$$




\subsection{Gelfand-Shilov spaces}

Let $h, \gamma, \tau>0$ be fixed. Then $S_{\tau ; h}^{\gamma}\left(\mathbb{R}^{d}\right)$ is the Banach space of all $f \in C^{\infty}\left(\mathbb{R}^{d}\right)$ such that

$$
\|f\|_{S_{\tau ; h}^{\gamma}}:=\sup _{p, q \in \mathbb{N}_{0}^{d}} \sup _{x \in \mathbb{R}^{d}} \frac{\left|x^{p} \partial^{q} f(x)\right|}{h^{|p|+|q|}|p| !^{\tau}|q| ! \gamma}<+\infty,
$$

endowed with the norm (6).

Definition 2.7 Let $\gamma, \tau>0$. The Gelfand-Shilov spaces $\mathcal{S}_{\tau}^{\gamma}\left(\mathbb{R}^{d}\right)$ and $\Sigma_{\tau}^{\gamma}\left(\mathbb{R}^{d}\right)$ are defined as unions and intersections of $S_{\tau ; h}^{\gamma}\left(\mathbb{R}^{d}\right)$ with respective inductive and projective limit topologies, respectively:

$$
\mathcal{S}_{\tau}^{\gamma}\left(\mathbb{R}^{d}\right):=\bigcup_{h>0} S_{\tau ; h}^{\gamma}\left(\mathbb{R}^{d}\right) \text { and } \Sigma_{\tau}^{\gamma}\left(\mathbb{R}^{d}\right):=\bigcap_{h>0} S_{\tau ; h}^{\gamma}\left(\mathbb{R}^{d}\right) .
$$

Note that $\Sigma_{\tau}^{\gamma}\left(\mathbb{R}^{d}\right) \neq\{0\}$ if and only if $\tau+\gamma \geq 1$ and $(\tau, \gamma) \neq(1 / 2,1 / 2)$, and $\mathcal{S}_{\tau}^{\gamma}\left(\mathbb{R}^{d}\right) \neq\{0\}$ if and only if $\tau+\gamma \geq 1$, see [22,35]. For every $\tau, \gamma, \varepsilon>0$ we have

$$
\Sigma_{\tau}^{\gamma}\left(\mathbb{R}^{d}\right) \hookrightarrow \mathcal{S}_{\tau}^{\gamma}\left(\mathbb{R}^{d}\right) \hookrightarrow \Sigma_{\tau+\varepsilon}^{\gamma+\varepsilon}\left(\mathbb{R}^{d}\right) \hookrightarrow \mathcal{S}\left(\mathbb{R}^{d}\right)
$$

If $\tau+\gamma \geq 1$, then the last two inclusions in (7) are dense, and if in addition $(\tau, \gamma) \neq$ $(1 / 2,1 / 2)$ then the first inclusion in (7) is dense. Moreover, for $\gamma<1$ the elements of $\mathcal{S}_{\tau}^{\gamma}\left(\mathbb{R}^{d}\right)$ can be extended to entire functions on $\mathbb{C}^{d}$ satisfying suitable exponential bounds [22].

In the sequel we will also use the following notation:

$$
\mathcal{S}^{(\gamma)}\left(\mathbb{R}^{d}\right):=\Sigma_{\gamma}^{\gamma}\left(\mathbb{R}^{d}\right), \quad \mathcal{S}^{\{\gamma\}}\left(\mathbb{R}^{d}\right):=\mathcal{S}_{\gamma}^{\gamma}\left(\mathbb{R}^{d}\right) \quad \text { and } \quad \mathcal{S}^{*}\left(\mathbb{R}^{d}\right)
$$

where $*$ stands for $(\gamma)$ or $\{\gamma\}$.

Definition 2.8 The Gelfand-Shilov distribution spaces $\left(\mathcal{S}_{\tau}^{\gamma}\right)^{\prime}\left(\mathbb{R}^{d}\right)$ and $\left(\Sigma_{\tau}^{\gamma}\right)^{\prime}\left(\mathbb{R}^{d}\right)$ are the projective and inductive limit respectively of $\left(S_{\tau ; h}^{\gamma}\right)^{\prime}\left(\mathbb{R}^{d}\right)$, the topological dual of $S_{\tau ; h}^{\gamma}\left(\mathbb{R}^{d}\right)$ :

$$
\left(\mathcal{S}_{\tau}^{\gamma}\right)^{\prime}\left(\mathbb{R}^{d}\right):=\bigcap_{h>0}\left(S_{\tau ; h}^{\gamma}\right)^{\prime}\left(\mathbb{R}^{d}\right) \text { and }\left(\Sigma_{\tau}^{\gamma}\right)^{\prime}\left(\mathbb{R}^{d}\right):=\bigcup_{h>0}\left(S_{\tau ; h}^{\gamma}\right)^{\prime}\left(\mathbb{R}^{d}\right)
$$

It follows that $\mathcal{S}^{\prime}\left(\mathbb{R}^{d}\right) \hookrightarrow\left(\mathcal{S}_{\tau}^{\gamma}\right)^{\prime}\left(\mathbb{R}^{d}\right)$ when $\tau+\gamma \geq 1$, and if in addition $(\tau, \gamma) \neq$ $(1 / 2,1 / 2)$, then $\left(\mathcal{S}_{\tau}^{\gamma}\right)^{\prime}\left(\mathbb{R}^{d}\right) \hookrightarrow\left(\Sigma_{\tau}^{\gamma}\right)^{\prime}\left(\mathbb{R}^{d}\right)$.

The Gelfand-Shilov spaces enjoy beautiful symmetric characterizations which also involve the Fourier transform of their elements. The following result has been reinvented several times, in similar or analogous terms, see [7,28,30,34].

Theorem 2.9 Let $\gamma, \tau \geq 1 / 2$. The following conditions are equivalent:

(i) $f \in \mathcal{S}_{\tau}^{\gamma}\left(\mathbb{R}^{d}\right)\left(\right.$ resp. $\left.f \in \Sigma_{\tau}^{\gamma}\left(\mathbb{R}^{d}\right)\right)$; 
(ii) There exist (resp. for every) constants $A, B>0$ such that

$$
\left\|x^{p} f(x)\right\|_{L^{\infty}} \lesssim A^{|p|}|p|^{!^{\tau}} \quad \text { and }\left\|\omega^{q} \hat{f}(\omega)\right\|_{L^{\infty}} \lesssim B^{|q|}|q| !^{\gamma}, \quad \forall p, q \in \mathbb{N}_{0}^{d}
$$

(iii) There exist (resp. for every) constants $A, B>0$ such that

$$
\left\|x^{p} f(x)\right\|_{L^{\infty}} \lesssim A^{|p|}|p| !^{\tau} \quad \text { and }\left\|\partial^{q} f(x)\right\|_{L^{\infty}} \lesssim B^{|q|}|q|^{!^{\gamma}}, \quad \forall p, q \in \mathbb{N}_{0}^{d}
$$

(iv) There exist (resp. for every) constants $h, k>0$ such that

$$
\left\|f(x) e^{h|x|^{1 / \tau}}\right\|_{L^{\infty}}<+\infty \text { and }\left\|\hat{f}(\omega) e^{k|\omega|^{1 / \gamma}}\right\|_{L^{\infty}}<+\infty
$$

(v) There exist (resp. for every) constants $h, B>0$ such that

$$
\left\|\left(\partial^{q} f\right)(x) e^{h|x|^{1 / \tau}}\right\|_{L^{\infty}} \lesssim B^{|q|}|q| !^{\gamma}, \quad \forall q \in \mathbb{N}_{0}^{d}
$$

Moreover, we could consider any $L^{p}$-norm, $1 \leq p<\infty$ instead of $L^{\infty}$-norm in Theorem 2.9, cf. [30].

By using Theorem 2.9 it can be shown that the Fourier transform is a topological isomorphism between $\mathcal{S}_{\tau}^{\gamma}\left(\mathbb{R}^{d}\right)$ and $\mathcal{S}_{\gamma}^{\tau}\left(\mathbb{R}^{d}\right), \gamma, \tau \geq 1 / 2\left(\mathcal{F}\left(\mathcal{S}_{\tau}^{\gamma}\right)\left(\mathbb{R}^{d}\right)=\mathcal{S}_{\gamma}^{\tau}\left(\mathbb{R}^{d}\right)\right)$, which extends to a continuous linear transform from $\left(\mathcal{S}_{\tau}^{\gamma}\right)^{\prime}\left(\mathbb{R}^{d}\right)$ onto $\left(\mathcal{S}_{\gamma}^{\tau}\right)^{\prime}\left(\mathbb{R}^{d}\right)$. Similar considerations hold for partial Fourier transforms with respect to some choice of variables. In particular, if $\gamma=\tau$ and $\gamma \geq 1 / 2$ then $\mathcal{F}\left(\mathcal{S}_{\gamma}^{\gamma}\right)\left(\mathbb{R}^{d}\right)=\mathcal{S}_{\gamma}^{\gamma}\left(\mathbb{R}^{d}\right)$, and if moreover $\gamma>1 / 2$, then $\mathcal{F}\left(\Sigma_{\gamma}^{\gamma}\right)\left(\mathbb{R}^{d}\right)=\Sigma_{\gamma}^{\gamma}\left(\mathbb{R}^{d}\right)$, and similarly for their distribution spaces. Due to this fact, corresponding dual spaces are referred to as tempered ultra-distributions (of Roumieu and Beurling type respectively), see [35].

The combination of global regularity with suitable decay properties at infinity [cf. (8)] which is built in the very definition of $\mathcal{S}_{\tau}^{\gamma}\left(\mathbb{R}^{d}\right)$ and $\Sigma_{\tau}^{\gamma}\left(\mathbb{R}^{d}\right)$, makes them suitable for the study of different problems in mathematical physics [22,23,34]. We refer to $[13,14,40,41]$ for the study of localization operators in the context of Gelfand-Shilov spaces. See also $[44,47,48]$ for related studies.

\subsection{Time-frequency representations}

In this subsection we recall the definitions and basic properties of the short-time Fourier transform and the (cross-) $\tau$-Wigner distribution.

Given a function $f$ on $\mathbb{R}^{d}$ and $x, \omega \in \mathbb{R}^{d}$, the translation operator $T_{x}$ and the modulation operator $M_{\omega}$ are defined as

$$
T_{x} f(t):=f(t-x) \text { and } M_{\omega} f(t):=e^{2 \pi i \omega t} f(t) \quad t \in \mathbb{R}
$$

and their composition $\pi(x, \omega):=M_{\omega} T_{x}$ is called time-frequency shift. We can now introduce two most commonly used time-frequency representations of a signal $f$, the so-called short-time Fourier transform (STFT) and the (cross-)Wigner distribution. 
Definition 2.10 Consider a window $g \in \mathcal{S}^{(1)}\left(\mathbb{R}^{d}\right) \backslash\{0\}$. The short-time Fourier transform of $f \in \mathcal{S}^{(1)}\left(\mathbb{R}^{d}\right)$ with respect to $g$ is the function defined on the phase-space as follows:

$$
V_{g} f(x, \omega):=\langle f, \pi(x, \omega) g\rangle=\int_{\mathbb{R}^{d}} \overline{g(t-x)} e^{-2 \pi i t \omega} f(t) d t, \quad(x, \omega) \in \mathbb{R}^{2 d} .
$$

We refer to Gröchenig [24, Chapter 3] for the properties and different equivalent forms of the STFT.

Definition 2.11 Let $\tau \in[0,1]$. The (cross-) $\tau$-Wigner distribution of $f, g \in \mathcal{S}^{(1)}\left(\mathbb{R}^{d}\right)$ is defined by

$$
W_{\tau}(f, g)(x, \omega):=\int_{\mathbb{R}^{d}} e^{-2 \pi i t \omega} f(x+\tau t) \overline{g(x-(1-\tau) t)} d t, \quad(x, \omega) \in \mathbb{R}^{2 d} .
$$

When $\tau=1 / 2, W_{1 / 2}(f, g)$ is simply called the cross-Wigner distribution of $f$ and $g$ and is denoted by $W(f, g)$ for short. Both STFT and $W_{\tau}$ are well defined for $f, g \in L^{2}\left(\mathbb{R}^{d}\right)$ and if the operator $\mathcal{A}_{\tau}, \tau \in(0,1)$, is defined on $L^{2}\left(\mathbb{R}^{d}\right)$ as

$$
\mathcal{A}_{\tau} f(t):=f\left(\frac{\tau-1}{\tau} t\right), \quad t \in \mathbb{R}^{d},
$$

then the connection between the STFT and $\tau$-Wigner distribution is described as follows.

Lemma 2.12 Let $g \in \mathcal{S}^{(1)}\left(\mathbb{R}^{d}\right) \backslash\{0\}$ and $f \in \mathcal{S}^{(1)}\left(\mathbb{R}^{d}\right)$.

(i) If $\tau \in(0,1)$, then

$$
W_{\tau}(f, g)(x, \omega)=\frac{1}{\tau^{d}} e^{2 \pi i \frac{1}{\tau} \omega x} V_{\mathcal{A}_{\tau} g} f\left(\frac{1}{1-\tau} x, \frac{1}{\tau} \omega\right), \quad \forall(x, \omega) \in \mathbb{R}^{2 d} ;
$$

(ii) if $\tau=0$, then

$$
W_{0}(f, g)(x, \omega)=e^{-2 \pi i x \omega} f(x) \overline{\hat{g}(\omega)}=R(f, g)(x, \omega), \quad \forall(x, \omega) \in \mathbb{R}^{2 d} ;
$$

(iii) if $\tau=1$, then

$$
W_{1}(f, g)(x, \omega)=e^{2 \pi i x \omega} \overline{g(x)} \hat{f}(\omega)=\overline{R(g, f)}(x, \omega), \quad \forall(x, \omega) \in \mathbb{R}^{2 d} ;
$$

where $R(f, g)$ denotes the Rihaczek distribution of $f$ and $g$.

Proof The proof is straightforward, and we show only $(i)$ for the sake of completeness (see also [15, Proposition 1.3.30]). After the change of variables $s=x+\tau t$ in (9) we obtain

$$
W_{\tau}(f, g)(x, \omega)=\frac{1}{\tau^{d}} \int_{\mathbb{R}^{d}} e^{-2 \pi i \frac{1}{\tau}(s-x) \omega} f(s) \overline{g\left(\frac{1}{\tau}(x-(1-\tau) s)\right)} d s
$$




$$
\begin{aligned}
& =\frac{1}{\tau^{d}} e^{2 \pi i \frac{1}{\tau} x \omega} \int_{\mathbb{R}^{d}} e^{-2 \pi i s \frac{\omega}{\tau}} f(s) \overline{\mathcal{A}_{\tau} g\left(s-\frac{x}{1-\tau}\right)} d s \\
& =\frac{1}{\tau^{d}} e^{2 \pi i \frac{1}{\tau} \omega x} V_{\mathcal{A}_{\tau} g} f\left(\frac{1}{1-\tau} x, \frac{1}{\tau} \omega\right), \quad \forall(x, \omega) \in \mathbb{R}^{2 d},
\end{aligned}
$$

since $\mathcal{A}_{\tau} g\left(s-\frac{x}{1-\tau}\right)=g\left(\frac{x}{\tau}+\frac{\tau-1}{\tau} s\right)$.

Notice that when $\tau=1 / 2$, we have $\mathcal{A}_{1 / 2} g(t)=g(-t)$, and (10) becomes

$$
W(f, g)(x, \omega)=2^{d} e^{4 \pi i x \cdot \omega} V_{\mathcal{A}_{1 / 2} g} f(2 x, 2 \omega), \quad \forall(x, \omega) \in \mathbb{R}^{2 d} .
$$

Definitions 2.10 and 2.11 are uniquely extended to $f \in\left(\mathcal{S}^{(1)}\right)^{\prime}\left(\mathbb{R}^{d}\right)$ by duality.

We will also use the following fact related to time-frequency representations of the Gelfand-Shilov spaces.

Theorem 2.13 Let $\mathcal{S}^{*}\left(\mathbb{R}^{d}\right)$ denote $\mathcal{S}^{\{\gamma\}}\left(\mathbb{R}^{d}\right), \gamma \geq 1 / 2$, or $\mathcal{S}^{(\gamma)}\left(\mathbb{R}^{d}\right), \gamma>1 / 2$. Moreover, let $g \in \mathcal{S}^{*}\left(\mathbb{R}^{d}\right) \backslash\{0\}$ and $\tau \in[0,1]$. Then the following are true:

(i) if $f \in \mathcal{S}^{*}\left(\mathbb{R}^{d}\right)$, then $W_{\tau}(f, g), V_{g} f \in \mathcal{S}^{*}\left(\mathbb{R}^{2 d}\right)$;

(ii) if $f \in\left(\mathcal{S}^{*}\right)^{\prime}\left(\mathbb{R}^{d}\right)$ and $W_{\tau}(f, g) \in \mathcal{S}^{*}\left(\mathbb{R}^{2 d}\right)$ or $V_{g} f \in \mathcal{S}^{*}\left(\mathbb{R}^{2 d}\right)$, then $f \in \mathcal{S}^{*}\left(\mathbb{R}^{d}\right)$.

Proof The proof for the STFT and $W_{1 / 2}$ can be found in several sources, see e.g. $[28,38,44]$. The case $\tau \in[0,1], \tau \neq 1 / 2$, can be proved in a similar fashion and is left for the reader as an exercise.

\subsection{Pseudodifferential and localization operators}

Next we introduce $\tau$-quantizations as pseudodifferential operators acting on $\mathcal{S}^{(1)}\left(\mathbb{R}^{d}\right)$. We address the reader to the textbooks $[15,24]$ in which the framework is mostly the one of $\mathcal{S}\left(\mathbb{R}^{d}\right)$ and $\mathcal{S}^{\prime}\left(\mathbb{R}^{d}\right)$, and we suggest [34,38,40,44,47,48] for the framework of Gelfand-Shilov spaces and their spaces of ultra-distributions.

Definition 2.14 Let $\tau \in[0,1]$. Given a symbol $\sigma \in\left(\mathcal{S}^{(1)}\right)^{\prime}\left(\mathbb{R}^{2 d}\right)$, the $\tau$-quantization of $\sigma$ is the pseudodifferential operator

$$
O p_{\tau}(\sigma): \mathcal{S}^{(1)}\left(\mathbb{R}^{d}\right) \rightarrow\left(\mathcal{S}^{(1)}\right)^{\prime}\left(\mathbb{R}^{d}\right)
$$

defined by the formal integral

$$
O p_{\tau}(\sigma) f(x):=\iint_{\mathbb{R}^{2 d}} e^{2 \pi i(x-y) \omega} \sigma((1-\tau) x+\tau y, \omega) f(y) d y d \omega,
$$

or, in a weak sense,

$$
\left\langle O p_{\tau}(\sigma) f, g\right\rangle=\int_{\mathbb{R}^{d}} \iint_{\mathbb{R}^{2 d}} e^{2 \pi i(x-y) \omega} \sigma((1-\tau) x+\tau y, \omega) f(y) \bar{g}(x) d y d \omega d x,
$$

$f, g \in \mathcal{S}^{(1)}\left(\mathbb{R}^{d}\right)$. 
The correspondence between the symbol $\sigma$ and the operator $O p_{\tau}(\sigma)$ given by (11) is known as the Shubin $\tau$-representation [37]. By a change of variables and an interchange of the order of integration, it can be shown that $O p_{\tau}(\sigma), \sigma \in\left(\mathcal{S}^{(1)}\right)^{\prime}\left(\mathbb{R}^{2 d}\right)$, and the (cross-) $\tau$-Wigner distribution are related by the following formula:

$$
\left\langle O p_{\tau}(\sigma) f, g\right\rangle=\left\langle\sigma, W_{\tau}(g, f)\right\rangle, \quad f, g \in \mathcal{S}^{(1)}\left(\mathbb{R}^{d}\right) .
$$

Thus, for $\tau=1 / 2$ (the Weyl quantization) we recover the Weyl pseudodifferential operators, and when $\tau=0$ we obtain the Kohn-Nirenberg operators. Commonly used equivalent notation for the Weyl operators in the literature are $\mathrm{Op}_{W}(\sigma), \mathrm{Op}^{w}(\sigma), L_{\sigma}$ or $\sigma^{w}$. The Weyl calculus reveals to be extremely important since every continuous and linear operator from $\mathcal{S}^{(1)}\left(\mathbb{R}^{d}\right)$ into $\left(\mathcal{S}^{(1)}\right)^{\prime}\left(\mathbb{R}^{d}\right)$ can be written as the Weyl transform of some (Weyl) symbol $\sigma \in\left(\mathcal{S}^{(1)}\right)^{\prime}\left(\mathbb{R}^{2 d}\right)$. This is due to the Schwartz kernel theorem when extended to the duality between $\mathcal{S}^{(1)}\left(\mathbb{R}^{d}\right)$ and $\left(\mathcal{S}^{(1)}\right)^{\prime}\left(\mathbb{R}^{d}\right)$, see $[32,40]$.

Next we introduce localization operators in the form of the STFT multipliers, and discuss their relation to $\tau$-quantizations given above.

Definition 2.15 Let there be given window functions $\varphi_{1}, \varphi_{2} \in \mathcal{S}^{(1)}\left(\mathbb{R}^{d}\right) \backslash\{0\}$ and a symbol $a \in\left(\mathcal{S}^{(1)}\right)^{\prime}\left(\mathbb{R}^{2 d}\right)$. Then the localization operator

$$
A_{a}^{\varphi_{1}, \varphi_{2}}: \mathcal{S}^{(1)}\left(\mathbb{R}^{d}\right) \rightarrow\left(\mathcal{S}^{(1)}\right)^{\prime}\left(\mathbb{R}^{d}\right)
$$

is the continuous and linear mapping formally defined by

$$
A_{a}^{\varphi_{1}, \varphi_{2}} f(t):=\iint_{\mathbb{R}^{2 d}} a(x, \omega) V_{\varphi_{1}} f(x, \omega) M_{\omega} T_{x} \varphi_{2}(t) d x d \omega
$$

or, in a weak sense,

$$
\left\langle A_{a}^{\varphi_{1}, \varphi_{2}} f, g\right\rangle:=\left\langle a, \overline{V_{\varphi_{1}} f} V_{\varphi_{2}} g\right\rangle, \quad f, g \in \mathcal{S}^{(1)}\left(\mathbb{R}^{d}\right) .
$$

It can be proved that every localization operator $A_{a}^{\varphi_{1}, \varphi_{2}}$ can be written in the Weyl form, i.e. identified with the Weyl pseudodifferential operator due to the following formula

$$
A_{a}^{\varphi_{1}, \varphi_{2}}=\mathrm{Op}_{1 / 2}\left(a * W\left(\varphi_{2}, \varphi_{1}\right)\right),
$$

and $\sigma=a * W\left(\varphi_{2}, \varphi_{1}\right)$ is called Weyl symbol of $A_{a}^{\varphi_{1}, \varphi_{2}}$. We refer to [5, Lemma 2.4] or [19] for the proof, see also [41].

By combining (12) and (14) we define $\tau$-localization operators as follows.

Let there be given $\tau \in[0,1]$, windows $\varphi_{1}, \varphi_{2} \in \mathcal{S}^{(1)}\left(\mathbb{R}^{d}\right) \backslash\{0\}$ and a symbol $a \in\left(\mathcal{S}^{(1)}\right)^{\prime}\left(\mathbb{R}^{2 d}\right)$. Then $\tau$-localization operator is defined to be

$$
A_{a, \tau}^{\varphi_{1}, \varphi_{2}}:=\mathrm{Op}_{\tau}\left(a * W_{\tau}\left(\varphi_{2}, \varphi_{1}\right)\right)
$$

In other words, every $\tau$-localization operator is identified with $\tau$-pseudodifferential operator associated to the symbol $\sigma_{\tau}=a * W_{\tau}\left(\varphi_{2}, \varphi_{1}\right)$. 
It turns out that the class of localization operators given by (15) coincides to the one given by Definition 2.15, see [45]. We give an independent proof based on the kernel argument, and point out that the result is essentially a restatement of (1.23) in [45].

Proposition 2.16 Let $\varphi_{1}, \varphi_{2} \in \mathcal{S}^{(1)}\left(\mathbb{R}^{d}\right) \backslash\{0\}, a \in\left(\mathcal{S}^{(1)}\right)^{\prime}\left(\mathbb{R}^{2 d}\right)$ and $\tau \in[0,1]$. Then

$$
A_{a}^{\varphi_{1}, \varphi_{2}}=A_{a, \tau}^{\varphi_{1}, \varphi_{2}}
$$

Proof By the Schwartz kernel theorem for $\mathcal{S}^{(1)}\left(\mathbb{R}^{d}\right)$ and $\left(\mathcal{S}^{(1)}\right)^{\prime}\left(\mathbb{R}^{d}\right)$, it suffices to show that the kernels of $A_{a}^{\varphi_{1}, \varphi_{2}}$ and $A_{a, \tau}^{\varphi_{1}, \varphi_{2}}$ coincide. From (13) it follows that

$$
\left\langle A_{a}^{\varphi_{1}, \varphi_{2}} f, g\right\rangle=\langle k, g \otimes \bar{f}\rangle
$$

where the kernel $k$ of the operator $A_{a}^{\varphi_{1}, \varphi_{2}}$ is given by

$$
k(t, y)=\iint_{\mathbb{R}^{2 d}} a(x, \omega) \overline{M_{\omega} T_{x} \varphi_{1}}(y) M_{\omega} T_{x} \varphi_{2}(t) d x d \omega .
$$

It remains to calculate the kernel of $A_{a, \tau}^{\varphi_{1}, \varphi_{2}}$. By the commutation relation $T_{x} M_{\omega}=$ $e^{-2 \pi i x \omega} M_{\omega} T_{x}$, and the covariance property of $\tau$-Wigner transform:

$$
W_{\tau}\left(T_{x} M_{\omega} f, T_{x} M_{\omega} g\right)(p, q)=W_{\tau}(f, g)(p-x, q-\omega)
$$

we calculate $a * W_{\tau}\left(\varphi_{2}, \varphi_{1}\right)$ and obtain

$$
\begin{aligned}
a * W_{\tau}\left(\varphi_{2}, \varphi_{1}\right)(p, q)= & \iint_{\mathbb{R}^{2 d}} a(x, \omega) W_{\tau}\left(T_{x} M_{\omega} \varphi_{2}, T_{x} M_{\omega} \varphi_{1}\right)(p, q) d x d \omega \\
= & \iint_{\mathbb{R}^{2 d}} a(x, \omega)\left(\int_{\mathbb{R}^{d}} M_{\omega} T_{x} \varphi_{2}(p+\tau s) \overline{M_{\omega} T_{x} \varphi_{1}}\right. \\
& \left.(p-(1-\tau) s) e^{-2 \pi i q s} d s\right) d x d \omega .
\end{aligned}
$$

Now by using a suitable interpretation of the oscillatory integrals in the distributional sense, and appropriate change of variables (cf. [41]) we get

$$
\begin{aligned}
& \left\langle\mathrm{Op}_{\tau}\left(a * W_{\tau}\left(\varphi_{2}, \varphi_{1}\right)\right) f, g\right\rangle=\left\langle a * W_{\tau}\left(\varphi_{2}, \varphi_{1}\right), W_{\tau}(g, f)\right\rangle \\
& =\iint_{\mathbb{R}^{2 d}} a(x, \omega) \iint_{\mathbb{R}^{2 d}}\left(\iint_{\mathbb{R}^{2 d}} M_{\omega} T_{x} \varphi_{2}(p+\tau s) \overline{M_{\omega} T_{x} \varphi_{1}}(p-(1-\tau) s) e^{-2 \pi i q(s-r)}\right. \\
& \quad \times \bar{g}(p+\tau r) f(p-(1-\tau) r) d s d r) d p d q d x d \omega \\
& =\iint_{\mathbb{R}^{2 d}} \iint_{\mathbb{R}^{2 d}} a(x, \omega) M_{\omega} T_{x} \varphi_{2}(t) \overline{M_{\omega} T_{x} \varphi_{1}}(y) d x d \omega \bar{g}(t) f(y) d t d y \\
& =\left\langle k_{\tau}, g \otimes \bar{f}\right\rangle=\langle k, g \otimes \bar{f}\rangle,
\end{aligned}
$$


where $k$ is given by (16). By the uniqueness of the kernel we conclude that

$$
A_{a}^{\varphi_{1}, \varphi_{2}}=A_{a, \tau}^{\varphi_{1}, \varphi_{2}}
$$

and the proof is finished.

\subsection{Ultra-modulation spaces}

We use the terminology ultra-modulation spaces in order to emphasize that such spaces may contain ultra-distributions, contrary to the most usual situation when members of modulation spaces are tempered distributions. However, ultra-modulation spaces belong to the family of modulation spaces introduced in [18]. We refer to e.g. [46,48] for a general approach to the broad class of modulation spaces.

Definition 2.17 Fix a non-zero window $g \in \mathcal{S}^{(1)}\left(\mathbb{R}^{d}\right)$, a weight $m \in \mathscr{P}_{E}\left(\mathbb{R}^{2 d}\right)$ and $0<p, q \leq \infty$. The ultra-modulation space $M_{m}^{p, q}\left(\mathbb{R}^{d}\right)$ consists of all tempered ultradistributions $f \in\left(\mathcal{S}^{(1)}\right)^{\prime}\left(\mathbb{R}^{d}\right)$ such that the (quasi-)norm

$$
\|f\|_{M_{m}^{p, q}}:=\left\|V_{g} f\right\|_{L_{m}^{p, q}}=\left(\int_{\mathbb{R}^{d}}\left(\int_{\mathbb{R}^{d}}\left|V_{g} f(x, \omega)\right|^{p} m(x, \omega)^{p} d x\right)^{\frac{q}{p}} d \omega\right)^{\frac{1}{q}}
$$

(obvious modifications with $p=\infty$ or $q=\infty$ ) is finite.

We write $M_{m}^{p}\left(\mathbb{R}^{d}\right)$ for $M_{m}^{p, p}\left(\mathbb{R}^{d}\right)$, and $M^{p, q}\left(\mathbb{R}^{d}\right)$ if $m \equiv 1$.

We recall that the spaces $M_{m}^{p, q}\left(\mathbb{R}^{d}\right) \subset \mathcal{S}^{\prime}\left(\mathbb{R}^{d}\right)$, with $1 \leq p, q \leq \infty, g \in \mathcal{S}\left(\mathbb{R}^{d}\right)$ and $m$ of at most polynomial growth at infinity, were invented by Feichtinger [18] and called modulation spaces. There it was proved that they are Banach spaces and that different window functions in $\mathcal{S}\left(\mathbb{R}^{d}\right) \backslash\{0\}$ yield equivalent norms. Moreover, the window class can be enlarged to the Feichtinger algebra $M_{v}^{1,1}\left(\mathbb{R}^{d}\right)$, where $v$ is a submultiplicative weight of at most polynomial growth at infinity such that $m$ is $v$-moderate.

It turned out that properties analogous to the Banach case hold in the quasi-Banach one as well, see [21]. Moreover, such properties remain valid also in the more general setting of Definition 2.17. We collect them in the following theorem in the same manner as it is done in e.g. [46,47], see also the references given there.

Theorem 2.18 Consider $0<p, p_{1}, p_{2}, q, q_{1}, q_{2} \leq \infty$ and weights $m, m_{1}, m_{2} \in$ $\mathscr{P}_{E}\left(\mathbb{R}^{2 d}\right)$. Let $\|\cdot\|_{M_{m}^{p, q}}$ be given by (17) for a fixed $g \in \mathcal{S}^{(1)}\left(\mathbb{R}^{d}\right) \backslash\{0\}$. Then:

(i) $\left(M_{m}^{p, q}\left(\mathbb{R}^{d}\right),\|\cdot\|_{M_{m}^{p, q}}\right)$ is a quasi-Banach space whenever at least one between $p$ and $q$ is strictly smaller than 1, otherwise it is a Banach space;

(ii) if $\tilde{g} \in \mathcal{S}^{(1)}\left(\mathbb{R}^{d}\right) \backslash\{0\}, \tilde{g} \neq g$, then it induces a (quasi-)norm equivalent to $\|\cdot\|_{M_{m}^{p, q}}$

(iii) if $p_{1} \leq p_{2}, q_{1} \leq q_{2}$ and $m_{2} \lesssim m_{1}$, then;

$$
\mathcal{S}^{(1)}\left(\mathbb{R}^{d}\right) \hookrightarrow M_{m_{1}}^{p_{1}, q_{1}}\left(\mathbb{R}^{d}\right) \hookrightarrow M_{m_{2}}^{p_{2}, q_{2}}\left(\mathbb{R}^{d}\right) \hookrightarrow\left(\mathcal{S}^{(1)}\right)^{\prime}\left(\mathbb{R}^{d}\right) ;
$$


(iv) if $p, q<\infty$, then :

$$
\left(M_{m}^{p, q}\right)^{\prime}\left(\mathbb{R}^{d}\right) \cong M_{1 / m}^{p^{\prime}, q^{\prime}}\left(\mathbb{R}^{d}\right)
$$

where

$$
p^{\prime}:=\left\{\begin{array}{lll}
\infty & \text { if } & 0<p \leq 1 \\
\frac{p}{p-1} & \text { if } & 1<p<\infty
\end{array}\right.
$$

and similarly for $q^{\prime}$.

Remark 2.19 Point (ii) of the previous theorem tell us that the definition of $M_{m}^{p, q}\left(\mathbb{R}^{d}\right)$ is independent of the choice of the window. Moreover, it can be shown that the class for window functions can be extended from $\mathcal{S}^{(1)}\left(\mathbb{R}^{d}\right)$ to $M_{v}^{r}\left(\mathbb{R}^{d}\right)$, where $r \leq p, p^{\prime}, q, q^{\prime}$ and $v \in \mathscr{P}_{E}\left(\mathbb{R}^{2 d}\right)$ is submultiplicative and such that $m$ is $v$-moderate [47].

We refer to Cordero [8] for the density of $\mathcal{S}^{(1)}\left(\mathbb{R}^{d}\right)$ in $M_{m}^{p, q}\left(\mathbb{R}^{d}\right)$.

The following proposition is proved in e.g. [39, Theorem 4.1], [44, Theorem 3.9].

Proposition 2.20 Consider $\gamma \geq 1$ and $1 \leq p, q \leq \infty$. Then

$$
\mathcal{S}^{(\gamma)}\left(\mathbb{R}^{d}\right)=\bigcap_{k \geq 0} M_{w_{k}^{\gamma}}^{p, q}\left(\mathbb{R}^{d}\right), \quad\left(\mathcal{S}^{(\gamma)}\right)^{\prime}\left(\mathbb{R}^{d}\right)=\bigcup_{k \geq 0} M_{1 / w_{k}^{\gamma}}^{p, q}\left(\mathbb{R}^{d}\right) .
$$

In some situations it is convenient to consider ultra-modulation spaces as subspaces of $\left(\mathcal{S}^{\{1 / 2\}}\right)^{\prime}\left(\mathbb{R}^{d}\right)$ (taking the window $g$ in $\mathcal{S}^{\{1 / 2\}}\left(\mathbb{R}^{d}\right)$ ), see for example $[8,47]$. However, for our purposes it is sufficient to consider the weights in $\mathscr{P}_{E}\left(\mathbb{R}^{2 d}\right)$, and then $M_{m}^{p, q}\left(\mathbb{R}^{d}\right)$ is a subspace of $\left(\mathcal{S}^{(1)}\right)^{\prime}\left(\mathbb{R}^{d}\right)$. We address the reader to Toft [47, Proposition 1.1] and references quoted there for more details.

We restate [13, Proposition 2.6] in a simplified case suitable to our purposes.

Proposition 2.21 Assume $1 \leq p, q \leq \infty, m \in \mathscr{P}_{E}\left(\mathbb{R}^{2 d}\right)$ and $g \in \mathcal{S}^{(1)}\left(\mathbb{R}^{d}\right)$ such that $\|g\|_{L^{2}}=1$. Then for every $f \in M_{m}^{p, q}\left(\mathbb{R}^{d}\right)$ the following inversion formula holds true:

$$
f=\iint_{\mathbb{R}^{2 d}} V_{g} f(x, \omega) M_{\omega} T_{x} g d x d \omega,
$$

where the equality holds in $M_{m}^{p, q}\left(\mathbb{R}^{d}\right)$.

The embeddings between modulation spaces are studied by many authors. We recall the recent contribution [29, Theorem 4.11], which is convenient for our purposes and which will be used in Lemma 3.5.

Theorem 2.22 Let $0<p_{j}, q_{j} \leq \infty, s_{j}, t_{j} \in \mathbb{R}$ for $j=1,2$ and consider the polynomial weights $v_{t_{j}}, v_{s_{j}}$ defined as in (4). Then

$$
M_{v_{t_{1}} \otimes v_{s_{1}}}^{p_{1}, q_{1}}\left(\mathbb{R}^{d}\right) \hookrightarrow M_{v_{t_{2}} \otimes v_{s_{2}}}^{p_{2}, q_{2}}\left(\mathbb{R}^{d}\right)
$$

if the following two conditions hold true: 
(i) $\left(p_{1}, p_{2}, t_{1}, t_{2}\right)$ satisfies one of the following conditions:

$$
\begin{aligned}
& \left(\mathcal{C}_{1}\right) \frac{1}{p_{2}} \leq \frac{1}{p_{1}}, \quad t_{2} \leq t_{1} \\
& \left(\mathcal{C}_{2}\right) \frac{1}{p_{2}}>\frac{1}{p_{1}}, \quad \frac{1}{p_{2}}+\frac{t_{2}}{d}<\frac{1}{p_{1}}+\frac{t_{1}}{d} ;
\end{aligned}
$$

(ii) $\left(q_{1}, q_{2}, s_{1}, s_{2}\right)$ satisfies one of the conditions $\left(\mathcal{C}_{1}\right)$ or $\left(\mathcal{C}_{2}\right)$ with $p_{j}$ and $t_{j}$ replaced by $q_{j}$ and $s_{j}$ respectively.

\subsection{Gabor frames}

Consider a lattice $\Lambda:=\alpha \mathbb{Z}^{d} \times \beta \mathbb{Z}^{d} \subset \mathbb{R}^{2 d}$ for some $\alpha, \beta>0$. Given $g \in L^{2}\left(\mathbb{R}^{d}\right) \backslash\{0\}$, the set of time-frequency shifts $\mathcal{G}(g, \Lambda):=\{\pi(\lambda) g: \lambda \in \Lambda\}$ is called a Gabor system. The set $\mathcal{G}(g, \Lambda)$ is a Gabor frame if there exist constants $A, B>0$ such that

$$
A\|f\|_{L^{2}}^{2} \leq \sum_{\lambda \in \Lambda}|\langle f, \pi(\lambda) g\rangle|^{2} \leq B\|f\|_{L^{2}}^{2}, \quad \forall f \in L^{2}\left(\mathbb{R}^{d}\right) .
$$

If $\mathcal{G}(g, \Lambda)$ is a Gabor frame, then the frame operator

$$
S f:=\sum_{\lambda \in \Lambda}\langle f, \pi(\lambda) g\rangle \pi(\lambda) g, \quad f \in L^{2}\left(\mathbb{R}^{d}\right),
$$

is a topological isomorphism on $L^{2}\left(\mathbb{R}^{d}\right)$. Moreover, if we define $h:=S^{-1} g \in L^{2}\left(\mathbb{R}^{d}\right)$, then the system $\mathcal{G}(h, \Lambda)$ is a Gabor frame and we have the reproducing formulae

$$
f=\sum_{\lambda \in \Lambda}\langle f, \pi(\lambda) g\rangle \pi(\lambda) h=\sum_{\lambda \in \Lambda}\langle f, \pi(\lambda) h\rangle \pi(\lambda) g, \quad \forall f \in L^{2}\left(\mathbb{R}^{d}\right),
$$

with unconditional convergence in $L^{2}\left(\mathbb{R}^{d}\right)$. The window $h$ is called the canonical dual window of $g$. In particular, if $h=g$ and $\|g\|_{L^{2}}=1$, then $A=B=1$, the frame operator is the identity on $L^{2}\left(\mathbb{R}^{d}\right)$ and the Gabor frame is called Parseval Gabor frame. In particular, from (19) we can recover exactly the $L^{2}$-norm of every vector:

$$
\|f\|_{L^{2}}^{2}=\sum_{\lambda \in \Lambda}|\langle f, \pi(\lambda) g\rangle|^{2}, \quad \forall f \in L^{2}\left(\mathbb{R}^{d}\right) .
$$

Any window $h \in L^{2}\left(\mathbb{R}^{d}\right)$ such that (20) is satisfied is called alternative dual window for $g$. Given two functions $g, h \in L^{2}\left(\mathbb{R}^{d}\right)$ we are able to extend the notion of Gabor frame operator to the operator $S_{g, h}=S_{g, h}^{\Lambda}$ in the following way:

$$
S_{g, h} f:=\sum_{\lambda \in \Lambda}\langle f, \pi(\lambda) g\rangle \pi(\lambda) h, \quad f \in L^{2}\left(\mathbb{R}^{d}\right),
$$


whenever this is well defined. With this notation the reproducing formulae (20) can be rephrased as $S_{g, h}=I=S_{h, g}$, where $I$ is the identity on $L^{2}\left(\mathbb{R}^{d}\right)$.

Discrete equivalent norms produced by means of Gabor frames make of ultramodulation spaces a natural framework for time-frequency analysis. We address the reader to Galperin and Samarah [21], Gröchenig [24], and Toft [46,47].

Theorem 2.23 Consider $m, v \in \mathscr{P}_{E}\left(\mathbb{R}^{2 d}\right)$ such that $v$ is submultiplicative and $m$ is $v$-moderate. Take $\Lambda:=\alpha \mathbb{Z}^{d} \times \beta \mathbb{Z}^{d}$, for some $\alpha, \beta>0$, and $g, h \in \mathcal{S}^{(1)}\left(\mathbb{R}^{d}\right)$ such that $S_{g, h}=I$ on $L^{2}\left(\mathbb{R}^{d}\right)$. Then

$$
f=\sum_{\lambda \in \Lambda}\langle f, \pi(\lambda) g\rangle \pi(\lambda) h=\sum_{\lambda \in \Lambda}\langle f, \pi(\lambda) h\rangle \pi(\lambda) g, \quad \forall f \in M_{m}^{p, q}\left(\mathbb{R}^{d}\right)
$$

with unconditional convergence in $M_{m}^{p, q}\left(\mathbb{R}^{d}\right)$ if $0<p, q<\infty$ and with weak-* convergence in $M_{1 / v}^{\infty}\left(\mathbb{R}^{d}\right)$ otherwise. Moreover, there exist $0<A \leq B$ such that, for every $f \in M_{m}^{p, q}\left(\mathbb{R}^{d}\right)$,

$$
A\|f\|_{M_{m}^{p, q}} \leq\left(\sum_{n \in \mathbb{Z}^{d}}\left(\sum_{k \in \mathbb{Z}^{d}}|\langle f, \pi(\alpha k, \beta n) g\rangle|^{p} m(\alpha k, \beta n)^{p}\right)^{\frac{q}{p}} \leq B\|f\|_{M_{m}^{p, q},}^{\frac{1}{q}}\right.
$$

independently of $p, q$, and $m$. Equivalently:

$$
\|f\|_{M_{m}^{p, q}\left(\mathbb{R}^{d}\right)} \asymp\left\|(\langle f, \pi(\lambda) g\rangle)_{\lambda}\right\|_{\ell_{m}^{p, q}(\Lambda)}=\left\|\left(V_{g} f(\lambda)\right)_{\lambda}\right\|_{\ell_{m}^{p, q}(\Lambda)} .
$$

Similar inequalities hold with g replaced by $h$.

Now we are able to prove the convolution relations for ultra-modulations spaces which will be used to prove our main results in Sect. 3. For the Banach cases with weight of at most polynomial growth at infinity, convolution relations were studied in e.g $[9,42,43]$. We modify the technique used in [2] to the Gelfand-Shilov framework presented so far. The essential tool is the equivalence between continuous and discrete norm (21).

Proposition 2.24 Let there be given $0<p, q, r, t, u, \gamma \leq \infty$ such that

$$
\frac{1}{u}+\frac{1}{t}=\frac{1}{\gamma}
$$

and

$$
\frac{1}{p}+\frac{1}{q}=1+\frac{1}{r}, \text { for } 1 \leq r \leq \infty
$$

whereas

$$
p=q=r, \quad \text { for } 0<r<1 \text {. }
$$


Consider $m, v, \mu \in \mathscr{P}_{E}\left(\mathbb{R}^{2 d}\right)$ such that $m$ is $v$-moderate. Then

$$
M_{\left.m\right|_{1} \otimes \mu}^{p, u}\left(\mathbb{R}^{d}\right) * M_{\left.\left.v\right|_{1} \otimes v\right|_{2} \mu^{-1}}^{q, t}\left(\mathbb{R}^{d}\right) \hookrightarrow M_{m}^{r, \gamma}\left(\mathbb{R}^{d}\right)
$$

where $\left.m\right|_{1},\left.v\right|_{1},\left.v\right|_{2}$ are defined as in (1).

Proof First observe that due to Lemmas 2.2 and 2.3 it follows that the ultra-modulation spaces which came into play are well defined.

The main tool is the idea contained in [9, Proposition 2.4]. We take the ultramodulation norm with respect to the Gaussian windows $g_{0}(x):=e^{-\pi x^{2}} \in \mathcal{S}^{\{1 / 2\}}\left(\mathbb{R}^{d}\right)$ and $g(x):=2^{-d / 2} e^{-\pi x^{2} / 2}=\left(g_{0} * g_{0}\right)(x) \in \mathcal{S}^{\{1 / 2\}}\left(\mathbb{R}^{d}\right)$.

Since the involution operator $g^{*}(x)=\overline{g(-x)}$ and the modulation operator $M_{\omega}$ commute, by a direct computation we have

$$
M_{\omega}\left(g_{0}^{*} * g_{0}^{*}\right)=M_{\omega} g_{0}^{*} * M_{\omega} g_{0}^{*}
$$

and

$$
V_{g} f(x, \omega)=e^{-2 \pi i x \omega}\left(f * M_{\omega} g^{*}\right)(x) .
$$

Thus, by using the associativity and commutativity of the convolution product, we obtain

$$
\begin{aligned}
& V_{g}(f * h)(x, \omega)=e^{-2 \pi i x \omega}\left((f * h) * M_{\omega} g^{*}\right)(x) \\
& =e^{-2 \pi i x \omega}\left(\left(f * M_{\omega} g_{0}^{*}\right) *\left(h * M_{\omega} g_{0}^{*}\right)\right)(x) .
\end{aligned}
$$

We use the norm equivalence (21) for a suitable $\Lambda=\alpha \mathbb{Z}^{d} \times \beta \mathbb{Z}^{d}$, and then the $v$-moderateness in order to majorize $m$ :

$$
m(\alpha k, \beta n) \lesssim m(\alpha k, 0) v(0, \beta n)=\left.\left.m\right|_{1}(\alpha k) v\right|_{2}(\beta n) .
$$

Eventually Young's convolution inequality for sequences is used in the $k$-variable and Hölder's one in the $n$-variable. Indeed both inequalities can be used since $p, q, r$, $\gamma, t, u$ fulfill the assumptions of the proposition. We write in details the case when $r, \gamma, t, u<\infty$ and leave to the reader the remaining cases, when one among the indices $r, \gamma, t, u$ is equal to $\infty$, which can be done analogously.

$$
\begin{array}{rl}
\| f & * h\left\|_{M_{m}^{r, \gamma}} \asymp\right\|\left(\left(V_{g}(f * h)\right)(\alpha k, \beta n) m(\alpha k, \beta n)\right)_{k, n} \|_{\ell^{r, \gamma}\left(\mathbb{Z}^{2 d}\right)} \\
& \lesssim\left(\left.\sum_{n \in \mathbb{Z}^{d}}\left(\left.\sum_{k \in \mathbb{Z}^{d}}\left|\left(f * M_{\beta n} g_{0}^{*}\right) *\left(h * M_{\beta n} g_{0}^{*}\right)(\alpha k)\right|^{r} m\right|_{1}(\alpha k)^{r}\right)^{\gamma / r} v\right|_{2}(\beta n)^{\gamma}\right)^{1 / \gamma} \\
& =\left(\left.\sum_{n \in \mathbb{Z}^{d}}\left\|\left(f * M_{\beta n} g_{0}^{*}\right) *\left(h * M_{\beta n} g_{0}^{*}\right)\right\|_{\ell_{\left.m\right|_{1}}^{r}\left(\alpha \mathbb{Z}^{d}\right)}^{\gamma} v\right|_{2}(\beta n)^{\gamma}\right)^{1 / \gamma}
\end{array}
$$




$$
\begin{aligned}
& \lesssim\left(\left.\sum_{n \in \mathbb{Z}^{d}}\left\|f * M_{\beta n} g_{0}^{*}\right\|_{\ell_{\left.m\right|_{1}}^{p}\left(\alpha \mathbb{Z}^{d}\right)}^{\gamma}\left\|h * M_{\beta n} g_{0}^{*}\right\|_{\ell_{\left.v\right|_{1}}^{q}\left(\alpha \mathbb{Z}^{d}\right)}^{\gamma} v\right|_{2}(\beta n)^{\gamma}\right)^{1 / \gamma} \\
& \lesssim\left(\sum_{n \in \mathbb{Z}^{d}}\left\|f * M_{\beta n} g_{0}^{*}\right\|_{\ell_{\left.m\right|_{1}}^{p}\left(\alpha \mathbb{Z}^{d}\right)}^{u} \mu(\beta n)^{u}\right)^{\frac{1}{u}}\left(\sum_{n \in \mathbb{Z}^{d}}\left\|h * M_{\beta n} g_{0}^{*}\right\|_{\ell_{\left.v\right|_{1}}^{q}\left(\alpha \mathbb{Z}^{d}\right)} \frac{\left.v\right|_{2}(\beta n)^{t}}{\mu(\beta n)^{t}}\right)^{\frac{1}{t}} \\
& =\left\|\left(\left(V_{g_{0}} f\right)(\lambda)\right)_{\lambda}\right\|_{\ell_{\left.m\right|_{1} \otimes \mu}^{p, u}(\Lambda)}\left\|\left(\left(V_{g_{0}} h\right)(\lambda)\right)_{\lambda}\right\|_{\ell_{\left.\left.v\right|_{1} \otimes v\right|_{2} \mu^{-1}}^{q, t}(\Lambda)} . \\
& \asymp\|f\|_{M_{\left.m\right|_{1} \otimes \mu}^{p, u}}\|h\|_{M_{\left.\left.v\right|_{1} \otimes v\right|_{2} \mu^{-1}}^{q, t}} \cdot
\end{aligned}
$$

This concludes the proof.

\section{Main results}

From the previous observations it follows that localization operators are in fact a particular class of pseudodifferential operators, cf. (14) and (15). Therefore, in the study of eigenfunctions of localization operators we first observe operators $O p_{\tau}(\sigma)$ when $\sigma$ belongs to weighted modulation space $M_{w}^{\infty, 1}\left(\mathbb{R}^{2 d}\right)$, where $w$ is of a certain subexponential growth at infinity.

We first prove some continuity properties of $O p_{\tau}(\sigma)$ on ultra-modulation spaces (Theorem 3.3), and then use this result to prove subexponential decay of eigenfunctions of certain $\tau$-pseudodifferential operators (Theorem 3.7). Finally, we conclude the paper with subexponential decay and regularity properties of eigenfunctions of localization operators in terms of Gelfand-Shilov spaces (Theorem 3.8).

An important relation between the action of an operator $O p_{\tau}(\sigma)$ on time-frequency shifts and the STFT of its symbol $\sigma$ is explained in [12]. The setting given there is the one of $\mathcal{S}\left(\mathbb{R}^{d}\right)$ and $\mathcal{S}^{\prime}\left(\mathbb{R}^{d}\right)$, but it is easy to see that the claim is still valid when dealing with $\mathcal{S}^{(1)}\left(\mathbb{R}^{d}\right)$ and $\left(\mathcal{S}^{(1)}\right)^{\prime}\left(\mathbb{R}^{d}\right)$. Moreover, $\mathcal{S}^{(1)}\left(\mathbb{R}^{d}\right)$ and its dual can be replaced by $\mathcal{S}^{\{\gamma\}}\left(\mathbb{R}^{d}\right)$ and $\mathcal{S}^{\{\gamma\}^{\prime}}\left(\mathbb{R}^{d}\right)$ as it is done in [11] when $\tau=1 / 2$. Thus, the proof of the following lemma is omitted, since it follows by a slight modification of the proof of [12, Lemma 4.1].

Lemma 3.1 Consider $\tau \in[0,1], g \in \mathcal{S}^{(1)}\left(\mathbb{R}^{d}\right), \Phi_{\tau}:=W_{\tau}(g, g) \in \mathcal{S}^{(1)}\left(\mathbb{R}^{2 d}\right)$. If $\sigma \in\left(\mathcal{S}^{(1)}\right)^{\prime}\left(\mathbb{R}^{2 d}\right)$, then

$$
\left|\left\langle O p_{\tau}(\sigma) \pi(z) g, \pi(w) g\right\rangle\right|=\left|V_{\Phi_{\tau}} \sigma\left(\mathcal{T}_{\tau}(w, z), J(w-z)\right)\right|, \quad \forall z, w \in \mathbb{R}^{2 d},
$$

where $z=\left(z_{1}, z_{2}\right), w=\left(w_{1}, w_{2}\right) \in \mathbb{R}^{2 d}$ and $\mathcal{T}_{\tau}$ and $J$ are defined as follows:

$$
\mathcal{T}_{\tau}(w, z):=\left((1-\tau) w_{1}+\tau z_{1}, \tau w_{2}+(1-\tau) z_{2}\right), \quad J(z):=\left(z_{2},-z_{1}\right) .
$$

The following lemma can be viewed as a form of the inversion formula (18). The independent proof is given in the Appendix. 
Lemma 3.2 Let $\tau \in[0,1]$ and $\sigma \in\left(\mathcal{S}^{(1)}\right)^{\prime}\left(\mathbb{R}^{2 d}\right)$. If $g \in \mathcal{S}^{(1)}\left(\mathbb{R}^{d}\right)$ with $\|g\|_{L^{2}}=1$ and $f \in \mathcal{S}^{(1)}\left(\mathbb{R}^{d}\right)$, then

$$
O p_{\tau}(\sigma) f=\int_{\mathbb{R}^{2 d}} V_{g} f(z) O p_{\tau}(\sigma)(\pi(z) g) d z
$$

in the sense that

$$
\left\langle O p_{\tau}(\sigma) f, \varphi\right\rangle=\int_{\mathbb{R}^{2 d}} V_{g} f(z)\left\langle O p_{\tau}(\sigma)(\pi(z) g), \varphi\right\rangle d z, \quad \forall \varphi \in \mathcal{S}^{(1)}\left(\mathbb{R}^{d}\right) .
$$

Next we show how the $\tau$-quantization $O p_{\tau}(\sigma), \tau \in[0,1]$, can be extended between ultra-modulation spaces under suitable assumptions on the weights. We remark that the following theorem is contained in more general [47, Theorem 3.1]. A more elementary proof of the same claim when Lebesgue parameters are greater than or equal to 1 is given in [45, Theorem A.2]. In contrast to $[45,47]$ we use different arguments. Namely, our proof is based on the Schur test in combination with Lemmas 3.1 and 3.2. We note that [2, Theorem 3.3] is a particular case of Theorem 3.3 when restricted to polynomial weights and the duality between $\mathcal{S}\left(\mathbb{R}^{d}\right)$ and $\mathcal{S}^{\prime}\left(\mathbb{R}^{d}\right)$.

Theorem 3.3 Consider $\tau \in[0,1], m_{0} \in \mathscr{P}_{E}\left(\mathbb{R}^{4 d}\right)$ and $m_{1}, m_{2} \in \mathscr{P}_{E}\left(\mathbb{R}^{2 d}\right)$ such that $\frac{m_{2}(x, \omega)}{m_{1}(y, \eta)} \lesssim m_{0}((1-\tau) x+\tau y, \tau \omega+(1-\tau) \eta, \omega-\eta, y-x), \quad \forall x, \omega, y, \eta \in \mathbb{R}^{d}$

Fix a symbol $\sigma \in M_{m_{0}}^{\infty, 1}\left(\mathbb{R}^{2 d}\right)$. Then the pseudodifferential operator $O p_{\tau}(\sigma)$, from $\mathcal{S}^{(1)}\left(\mathbb{R}^{d}\right)$ to $\left(\mathcal{S}^{(1)}\right)^{\prime}\left(\mathbb{R}^{d}\right)$, extends uniquely to a bounded and linear operator from $M_{m_{1}}^{p}\left(\mathbb{R}^{d}\right)$ into $M_{m_{2}}^{p}\left(\mathbb{R}^{d}\right)$ for every $1 \leq p<\infty$.

Proof Let $g \in \mathcal{S}^{(1)}\left(\mathbb{R}^{d}\right)$ with $\|g\|_{L^{2}}=1$ and consider $f \in \mathcal{S}^{(1)}\left(\mathbb{R}^{d}\right) \subset M_{m_{1}}^{p}\left(\mathbb{R}^{d}\right)$. Due to the normalization chosen $\|g\|_{L^{2}}=\|\hat{g}\|_{L^{2}}$ and we recall the inversion formula (18) which can be seen as a pointwise equality between smooth functions in this case (see [24, Proposition 11.2.4]): $f=\int_{\mathbb{R}^{2 d}} V_{g} f(z) \pi(z) g d z$.

By Lemma 3.2 we have

$$
\begin{aligned}
V_{g}\left(O p_{\tau}(\sigma) f\right)(w) & =\left\langle O p_{\tau}(\sigma) f, \pi(w) g\right\rangle \\
& =\int_{\mathbb{R}^{2 d}} V_{g} f(z)\left\langle O p_{\tau}(\sigma) \pi(z) g, \pi(w) g\right\rangle d z
\end{aligned}
$$

In the next step we prove that the map $M_{\tau}(\sigma): G \mapsto M_{\tau}(\sigma) G$, defined by

$$
M_{\tau}(\sigma) G(w):=\int_{\mathbb{R}^{2 d}} G(z)\left\langle O p_{\tau}(\sigma) \pi(z) g, \pi(w) g\right\rangle d z
$$

is continuous from $L_{m_{1}}^{p}\left(\mathbb{R}^{2 d}\right)$ to $L_{m_{2}}^{p}\left(\mathbb{R}^{2 d}\right)$. 
Using (22), we see that it is equivalent to prove that the integral operator with kernel

$$
K_{\tau}(z, w):=\left|V_{\Phi_{\tau}} \sigma\left(\mathcal{T}_{\tau}(w, z), J(w-z)\right)\right| \frac{1}{m_{1}(z)} m_{2}(w)
$$

where $\mathcal{T}_{\tau}$ ans $J$ are defined in (23), is bounded on $L^{p}\left(\mathbb{R}^{2 d}\right)$. We do this using the Schur test (see, e.g., [24, Lemma 6.2.1 (b)]). First we majorize $K_{\tau}$ with another integral kernel $Q_{\tau}$ using the condition (25) with $w=(x, \omega) \in \mathbb{R}^{2 d}$ and $z=(y, \eta) \in \mathbb{R}^{2 d}$ :

$$
\begin{aligned}
K_{\tau}(z, w) & =\frac{m_{2}(w) m_{0}\left(\mathcal{T}_{\tau}(w, z), J(w-z)\right)}{m_{1}(z) m_{0}\left(\mathcal{T}_{\tau}(w, z), J(w-z)\right)}\left|V_{\Phi_{\tau}} \sigma\left(\mathcal{T}_{\tau}(w, z), J(w-z)\right)\right| \\
& \lesssim\left|V_{\Phi_{\tau}} \sigma\left(\mathcal{T}_{\tau}(w, z), J(w-z)\right)\right| m_{0}\left(\mathcal{T}_{\tau}(w, z), J(w-z)\right) \\
& =: Q_{\tau}(z, w) .
\end{aligned}
$$

We now show that $Q_{\tau}$ satisfies the Schur conditions. By appropriate change of variables $\left(w^{\prime} \equiv w_{z}^{\prime}(w):=J(w-z)\right.$, where $z$ is fixed) we obtain

$$
\begin{aligned}
\sup _{z \in \mathbb{R}^{2 d}} \int_{\mathbb{R}^{2 d}}\left|Q_{\tau}(z, w)\right| d w & =\int_{\mathbb{R}^{2 d}} \sup _{z \in \mathbb{R}^{2 d}}\left|V_{\Phi} \sigma\left(z, w^{\prime}\right)\right| m_{0}\left(z, w^{\prime}\right) d w^{\prime} \\
& =\|\sigma\|_{M_{m_{0}}^{\infty, 1}}<+\infty
\end{aligned}
$$

Furthermore, by the change of variables $w^{\prime} \equiv w_{w}^{\prime}(z):=J(w-z)$ for every $w$ fixed, we obtain

$$
\begin{aligned}
\sup _{w \in \mathbb{R}^{2 d}} \int_{\mathbb{R}^{2 d}}\left|Q_{\tau}(z, w)\right| d z & =\int_{\mathbb{R}^{2 d}} \sup _{w \in \mathbb{R}^{2 d}}\left|V_{\Phi_{\tau}} \sigma\left(w, w^{\prime}\right)\right| m_{0}\left(w, w^{\prime}\right) d w^{\prime} \\
& =\|\sigma\|_{M_{m_{0}}^{\infty, 1}}^{\infty}<+\infty
\end{aligned}
$$

Since $K_{\tau} \lesssim Q_{\tau}$, it follows that

$$
\sup _{z \in \mathbb{R}^{2 d}} \int_{\mathbb{R}^{2 d}}\left|K_{\tau}(z, w)\right| d w<+\infty \text { and } \sup _{w \in \mathbb{R}^{2 d}} \int_{\mathbb{R}^{2 d}}\left|K_{\tau}(z, w)\right| d z<+\infty \text {. }
$$

Hence from the Schur test it follows that $M_{\tau}(\sigma)$ is continuous, and due to (26) we notice that

$$
V_{g} \circ O p_{\tau}(\sigma) f=M_{\tau}(\sigma) \circ V_{g} f,
$$

where the right hand-side is continuous and takes elements of $\mathcal{S}^{(1)}\left(\mathbb{R}^{d}\right) \subset M_{m_{1}}^{p}\left(\mathbb{R}^{d}\right)$ into $L_{m_{2}}^{p}\left(\mathbb{R}^{2 d}\right)$. Therefore $O p_{\tau}(\sigma)$ is linear, continuous and densely defined. This concludes the proof.

Schatten class properties for various classes of pseudodifferential operators in the framework of time-frequency analysis are studied by many authors, let us mention just $[10,24,33,47]$. However, for our purposes it is convenient to recall [31, Theorem 1.2] 
about Schatten class property for pseudodifferential operators $O p_{\tau}(\sigma)$ with symbols in modulation spaces.

Theorem 3.4 Let $\tau \in[0,1], 0<p<2, d \in \mathbb{N}$ and

$$
u>\frac{2 d}{p}-d .
$$

Consider $\sigma \in M_{m_{u}^{\tau}}^{2}\left(\mathbb{R}^{2 d}\right)$, where $m_{u}^{\tau}$ is defined as in (5). Then

$$
O p_{\tau}(\sigma) \in S_{p}\left(L^{2}\left(\mathbb{R}^{d}\right)\right)
$$

Lemma 3.5 Let $\tau \in[0,1], \gamma \geq 1$ and $d \in \mathbb{N}$. Fix

$$
u, s, t>0, \quad l>u+d, \quad j \geq u .
$$

Then

$$
M_{w_{s}^{\gamma} \otimes w_{t}^{\gamma}}^{\infty, 1}\left(\mathbb{R}^{2 d}\right) \hookrightarrow M_{v_{l} \otimes v_{j}}^{\infty, 1}\left(\mathbb{R}^{2 d}\right) \hookrightarrow M_{v_{u} \otimes v_{u}}^{2}\left(\mathbb{R}^{2 d}\right) \hookrightarrow M_{m_{u}^{\tau}}^{2}\left(\mathbb{R}^{2 d}\right) .
$$

Proof The first inclusion is due to the inclusion relations between ultra-modulation spaces since $v_{l} \otimes v_{j} \lesssim w_{s}^{\gamma} \otimes w_{t}^{\gamma}$. The last inclusion follows similarly since $m_{u}^{\tau} \lesssim$ $v_{u} \otimes v_{u}$, as it is shown in Remark 2.5.

For the second inclusion we use Theorem 2.22: $(\infty, 2, l, u)$ fulfils the condition $\left(\mathcal{C}_{2}\right)$ and $(1,2, j, u)$ fulfils the condition $\left(\mathcal{C}_{1}\right)$. This concludes the proof.

On account of the following corollary all the operators considered in Theorem 3.7 are compact on $L^{2}\left(\mathbb{R}^{d}\right)$.

Corollary 3.6 Let $\tau \in[0,1], \gamma \geq 1$ and $s, t>0$. Consider $\sigma \in M_{w_{s}^{\gamma} \otimes w_{t}^{\gamma}}^{\infty, 1}\left(\mathbb{R}^{2 d}\right)$. Then $O p_{\tau}(\sigma)$ is compact on $L^{2}\left(\mathbb{R}^{d}\right)$.

Proof The claim follows by Lemma 3.5 with $u$ satisfying (27), after choosing any $0<p<2$, in addition with Theorem 3.4.

Now we prove the decay property of the eigenfunctions of $O p_{\tau}(\sigma)$ when the symbol belongs to certain weighted modulation spaces. This result improves [2, Proposition 3.6], in the sense that we show how faster decay of the symbol implies stronger regularity and decay properties for the eigenfunctions of the corresponding operator. More precisely, [2, Proposition 3.6] deals with polynomial decay, whereas Theorem 3.7 allows to consider sub-exponential decay as well.

Theorem 3.7 Fix $\tau \in[0,1], \gamma \geq 1$ and $s>0$. Consider a symbol $\sigma \in M_{w_{s}^{\gamma} \otimes w_{t}^{\gamma}}^{\infty, 1}\left(\mathbb{R}^{2 d}\right)$ for every $t \geq 0$.

If $\lambda \in \sigma_{P}\left(O p_{\tau}(\sigma)\right)$, then any $f \in L^{2}\left(\mathbb{R}^{d}\right)$ eigenfunction associated to the eigenvalue $\lambda$ belongs to $\mathcal{S}^{(\gamma)}\left(\mathbb{R}^{d}\right)$. 
Proof We first observe that $\sigma \in M_{w_{s}^{\gamma} \otimes w_{t}^{\gamma}}^{\infty, 1}\left(\mathbb{R}^{2 d}\right)$ for every $t \geq 0$ is equivalent to require that $t$ fulfills (2) due to the inclusion relations. By (3) from Lemma 2.4 it follows that

$$
\frac{w_{r^{\prime}+s^{\prime}}^{\gamma}(x, \omega)}{w_{r^{\prime}}^{\gamma}(y, \eta)} \leq w_{s^{\prime}}^{\gamma} \otimes w_{t^{\prime}}^{\gamma}(((1-\tau) x+\tau y, \tau \omega+(1-\tau) \eta),(\omega-\eta, y-x)),
$$

for every $x, \omega, y, \eta \in \mathbb{R}^{d}$, where $s^{\prime}, r^{\prime} \geq 0$ and $t^{\prime}$ which fulfils (2). We consider first the case $1 / 2 \leq \tau \leq 1$ and fix $s^{\prime}=s>0$.

Take $r^{\prime}=0, t \geq s \tau^{1 / \gamma}$, and apply Theorem 3.3 with $p=2, m_{0}=w_{s}^{\gamma} \otimes w_{t}^{\gamma}$, $m_{1}=w_{0}^{\gamma}$ and $m_{2}=w_{s}^{\gamma}$ which satisfy (25). Thus $O p_{\tau}(\sigma)$ extends to a continuous operator from $M_{w_{0}^{\gamma}}^{2}\left(\mathbb{R}^{d}\right)=L^{2}\left(\mathbb{R}^{d}\right)$ to $M_{w_{s}^{\gamma}}^{2}\left(\mathbb{R}^{d}\right)$. Starting with $f \in L^{2}\left(\mathbb{R}^{d}\right)$ we get $f=\lambda^{-1} O p_{\tau}(\sigma) f \in M_{w_{s}^{\gamma}}^{2}\left(\mathbb{R}^{d}\right)$.

Now, take $r^{\prime}=s, t \geq s+s \tau^{1 / \gamma}$, and apply Theorem 3.3 with $p=2, m_{0}=$ $w_{s}^{\gamma} \otimes w_{t}^{\gamma}, m_{1}=w_{s}^{\gamma}$ and $m_{2}=w_{2 s}^{\gamma}$ which satisfy (25). Thus $O p_{\tau}(\sigma)$ restricts to a continuous operator from $M_{w_{s}^{\gamma}}^{2}\left(\mathbb{R}^{d}\right)$ to $M_{w_{2 s}^{\gamma}}^{2}\left(\mathbb{R}^{d}\right)$, so starting with $f \in M_{w_{s}^{\gamma}}^{2}\left(\mathbb{R}^{d}\right)$ we get $f=\lambda^{-1} O p_{\tau}(\sigma) f \in M_{w_{2 s}^{\gamma}}^{2}\left(\mathbb{R}^{d}\right)$.

Repeating the same argument, and using the inclusion relations between ultramodulation spaces we obtain:

$$
f \in \bigcap_{n \in \mathbb{N}_{0}} M_{w_{n s}^{\gamma}}^{2}\left(\mathbb{R}^{d}\right)=\bigcap_{k \geq 0} M_{w_{k}^{\gamma}}^{2}\left(\mathbb{R}^{d}\right)=\mathcal{S}^{(\gamma)}\left(\mathbb{R}^{d}\right)
$$

The case $0 \leq \tau<1 / 2$ is done similarly. This concludes the proof.

We finish the paper with an observation related to localization operators.

Note that by Corollary 3.6 it follows that the localization operators $A_{a}^{\varphi_{1}, \varphi_{2}}$ in the following statement are compact on $L^{2}\left(\mathbb{R}^{d}\right)$.

Theorem 3.8 Consider $\gamma \geq 1, s>0, a \in M_{w_{s}^{\gamma} \otimes 1}^{\infty}\left(\mathbb{R}^{2 d}\right)$ and $\varphi_{1}, \varphi_{2} \in \mathcal{S}^{(1)}\left(\mathbb{R}^{d}\right)$. If $\lambda \in \sigma_{P}\left(A_{a}^{\varphi_{1}, \varphi_{2}}\right)$, then any $f \in L^{2}\left(\mathbb{R}^{d}\right)$ eigenfunction associated to the eigenvalue $\lambda$ belongs to $\mathcal{S}^{(\gamma)}\left(\mathbb{R}^{d}\right)$.

Proof Since $\varphi_{1}, \varphi_{2} \in \mathcal{S}^{(1)}\left(\mathbb{R}^{d}\right)$ it follows that $W\left(\varphi_{2}, \varphi_{1}\right) \in \mathcal{S}^{(1)}\left(\mathbb{R}^{2 d}\right) \subset$ $M_{w_{r}^{\gamma} \otimes w_{t}^{\gamma}}^{1}\left(\mathbb{R}^{2 d}\right)$, for every $r, t \geq 0$. It is easy to check that $w_{s}^{\gamma} \otimes w_{t}^{\gamma}$ is $w_{r}^{\gamma} \otimes w_{t}^{\gamma}$ moderate for every $t \geq 0$ and every $r \geq s$, i.e.

$$
w_{s}^{\gamma} \otimes w_{t}^{\gamma}((x, \omega)+(y, \eta)) \leq w_{r}^{\gamma} \otimes w_{t}^{\gamma}(x, \omega) w_{s}^{\gamma} \otimes w_{t}^{\gamma}(y, \eta), \quad x, \omega, y, \eta \in \mathbb{R}^{d} .
$$

We write $A_{a}^{\varphi_{1}, \varphi_{2}}=\mathrm{Op}^{w}(\sigma)$ with $\sigma=a * W\left(\varphi_{2}, \varphi_{1}\right)$, and then apply Proposition 2.24 in order to infer $\sigma \in M_{w_{s}^{\gamma} \otimes w_{t}^{\gamma}}^{\infty, 1}\left(\mathbb{R}^{2 d}\right)$ for every $t \geq s / 2^{1 / \gamma}$ :

$$
M_{w_{s}^{\gamma} \otimes 1}^{\infty}\left(\mathbb{R}^{2 d}\right) * M_{w_{r}^{\gamma} \otimes w_{t}^{\gamma}}^{1}\left(\mathbb{R}^{2 d}\right) \hookrightarrow M_{w_{s}^{\gamma} \otimes w_{t}^{\gamma}}^{\infty, 1}\left(\mathbb{R}^{2 d}\right) .
$$

The claim now follows by Theorem 3.7. 
Acknowledgements The authors would like to thank Elena Cordero and Fabio Nicola for fruitful conversations and comments. F. Bastianoni is member of the Gruppo Nazionale per l'Analisi Matematica, la Probabilità e le loro Applicazioni (GNAMPA) of the Istituto Nazionale di Alta Matematica (INdAM). N. Teofanov was partially supported by project ANACRES, MPNTR Grant No. 451-03-68/2020-14/200125, and Project 19.032/961-103/19 MNRVOID of the Republic of Srpska.

Funding Open access funding provided by Politecnico di Torino.

Open Access This article is licensed under a Creative Commons Attribution 4.0 International License, which permits use, sharing, adaptation, distribution and reproduction in any medium or format, as long as you give appropriate credit to the original author(s) and the source, provide a link to the Creative Commons licence, and indicate if changes were made. The images or other third party material in this article are included in the article's Creative Commons licence, unless indicated otherwise in a credit line to the material. If material is not included in the article's Creative Commons licence and your intended use is not permitted by statutory regulation or exceeds the permitted use, you will need to obtain permission directly from the copyright holder. To view a copy of this licence, visit http://creativecommons.org/licenses/by/4.0/.

\section{Appendix}

In this section we prove Lemmas 2.4 and 3.2.

Proof of Lemma 2.4 We first recall that given $0<p \leq q<\infty$ the following holds true

$$
\|z\|_{q}=\left(\sum_{i=1}^{d}\left|z_{i}\right|^{q}\right)^{\frac{1}{q}} \leq\left(\sum_{i=1}^{d}\left|z_{i}\right|^{p}\right)^{\frac{1}{p}}=\|z\|_{p}, \quad z=\left(z_{1}, \ldots, z_{d}\right) \in \mathbb{R}^{d}
$$

In fact, consider $z$ such that $\|z\|_{p}=1$. Hence $\left|z_{i}\right|^{p} \leq 1 \Rightarrow\left|z_{i}\right| \leq 1$ for $i=$ $1, \ldots, d$. Thus $\left|z_{i}\right|^{q} \leq\left|z_{i}\right|^{p}$ and $\sum_{i=1}^{d}\left|z_{i}\right|^{q} \leq \sum_{i=1}^{d}\left|z_{i}\right|^{p}=1$. Eventually consider $u \in \mathbb{R}^{d} \backslash\{0\}$, then $\|u /\| u\left\|_{p}\right\|_{q} \leq 1$ and (28) is proved.

By using the triangular inequality and (28) with $q=1$ and $p=\beta$, we infer that

$$
\left|\sum_{i=1}^{d} z_{i}\right|^{\beta} \leq \sum_{i=1}^{d}\left|z_{i}\right|^{\beta}, \quad z=\left(z_{1}, \ldots, z_{d}\right) \in \mathbb{R}^{d},
$$

for $0<\beta \leq 1$. Now, by the triangular inequality and (29) with $d=2$ we obtain

$$
|x|^{\beta}-|y|^{\beta} \leq|x-y|^{\beta}, \quad 0<\beta \leq 1, \quad x, y \in \mathbb{R}^{d} .
$$

Next, we observe that for $z, w \in \mathbb{R}^{d}$

$$
\begin{aligned}
& |(\tau z,(1-\tau) w)|^{2}=\tau^{2}|(z, w)|^{2}+(1-2 \tau)|w|^{2} \\
& \quad \leq \begin{cases}\tau^{2}|(z, w)|^{2} & \text { if } 1 / 2 \leq \tau \leq 1, \\
\tau^{2}|(z, w)|^{2}+1|w|^{2}+|z|^{2}=\left(1+\tau^{2}\right)|(z, w)|^{2} & \text { if } 0 \leq \tau<1 / 2,\end{cases}
\end{aligned}
$$


which gives

$$
|(\tau z,(1-\tau) w)|^{1 / \gamma} \leq \begin{cases}\tau^{1 / \gamma}|(z, w)|^{1 / \gamma} & \text { if } 1 / 2 \leq \tau \leq 1 \\ \left(1+\tau^{2}\right)^{1 / 2 \gamma}|(z, w)|^{1 / \gamma} & \text { if } 0 \leq \tau<1 / 2\end{cases}
$$

We can now prove (3):

$$
\begin{aligned}
\frac{w_{r+s}^{\gamma}(x, \omega)}{w_{r}^{\gamma}(y, \eta)} \stackrel{(30)}{\leq} \exp \left(r|(x, \omega)-(y, \eta)|^{1 / \gamma}+s|(x, \omega)|^{1 / \gamma}\right) \\
\quad \stackrel{(30)}{\leq} \exp \left(r|(\omega-\eta, y-x)|^{1 / \gamma}+s|(x, \omega)-(\tau(x-y),(1-\tau)(\omega-\eta))|^{1 / \gamma}\right. \\
\left.+\quad s|(\tau(x-y),(1-\tau)(\omega-\eta))|^{1 / \gamma}\right) \\
\stackrel{(31)}{\leq}\left\{\begin{array}{c}
\exp \left(\left(r+s \tau^{1 / \gamma}\right)|(\omega-\eta, y-x)|^{1 / \gamma}\right. \\
\left.+s|((1-\tau) x+\tau y, \tau \omega+(1-\tau) \eta)|^{1 / \gamma}\right) \quad \text { if } 1 / 2 \leq \tau \leq 1, \\
\exp \left(\left(r+s\left(1+\tau^{2}\right)^{1 / 2 \gamma}\right)|(\omega-\eta, y-x)|^{1 / \gamma}\right. \\
\left.+s|((1-\tau) x+\tau y, \tau \omega+(1-\tau) \eta)|^{1 / \gamma}\right)
\end{array} \quad \text { if } 0 \leq \tau<1 / 2,\right.
\end{aligned}
$$

and Lemma 2.4 follows from the assumption (2).

Proof of Lemma 3.2 Consider $\tau \in(0,1)$ and recast the $\tau$-Winger distribution $W_{\tau}(\varphi, f)$ using the operator $\mathcal{A}_{\tau} f(t):=f\left(\frac{\tau-1}{\tau} t\right)$ :

$$
\begin{aligned}
W_{\tau}(\varphi, f)(x, \omega) & =\frac{1}{\tau^{d}} e^{2 \pi i \frac{1}{\tau} \omega x} V_{\mathcal{A}_{\tau} f} \varphi\left(\frac{1}{1-\tau} x, \frac{1}{\tau} \omega\right) \\
& =\frac{1}{\tau^{d}} e^{2 \pi i \frac{1}{\tau} \omega x}\left\langle\varphi, M_{\frac{1}{\tau} \omega} T \frac{1}{1-\tau} x \mathcal{A}_{\tau} f\right\rangle \\
& =\frac{1}{\tau^{d}} e^{2 \pi i \frac{1}{\tau} \omega x}\left\langle\left(\frac{\tau}{1-\tau}\right)^{d} \mathcal{A}_{1-\tau} T_{-\frac{1}{1-\tau} x} M_{-\frac{1}{\tau} \omega} \varphi, f\right\rangle \\
& =\frac{1}{\tau^{d}} e^{2 \pi i \frac{1}{\tau} \omega x} \int_{\mathbb{R}^{2 d}} \overline{V_{g} f(z)}\left\langle\left(\frac{\tau}{1-\tau}\right)^{d} \mathcal{A}_{1-\tau} T_{-\frac{1}{1-\tau} x} M_{-\frac{1}{\tau} \omega} \varphi, \pi(z) g\right\rangle d z \\
& =\int_{\mathbb{R}^{2 d}} \overline{V_{g} f(z)} \frac{1}{\tau^{d}} e^{2 \pi i \frac{1}{\tau} \omega x}\left\langle\varphi, M_{\frac{1}{\tau} \omega} T_{\frac{1}{1-\tau} x} \mathcal{A}_{\tau} \pi(z) g\right\rangle d z \\
& =\int_{\mathbb{R}^{2 d}} \overline{V_{g} f(z)} \frac{1}{\tau^{d}} e^{2 \pi i \frac{1}{\tau} \omega x} V_{\mathcal{A}_{\tau} \pi(z) g} \varphi\left(\frac{1}{1-\tau} x, \frac{1}{\tau} \omega\right) d z \\
& =\int_{\mathbb{R}^{2 d}} \overline{V_{g} f(z)} W_{\tau}(\varphi, \pi(z) g)(x, \omega) d z .
\end{aligned}
$$

Therefore

$$
\begin{aligned}
\left\langle O p_{\tau}(\sigma) f, \varphi\right\rangle & =\left\langle\sigma, W_{\tau}(\varphi, f)\right\rangle \\
& =\left\langle\sigma, \int_{\mathbb{R}^{2 d}} \overline{V_{g} f(z)} W_{\tau}(\varphi, \pi(z) g)(x, \omega) d z\right\rangle \\
& =\int_{\mathbb{R}^{2 d}} V_{g} f(z)\left\langle\sigma, W_{\tau}(\varphi, \pi(z) g)(x, \omega)\right\rangle d z
\end{aligned}
$$




$$
=\int_{\mathbb{R}^{2 d}} V_{g} f(z)\left\langle O p_{\tau}(\sigma)(\pi(z) g), \varphi\right\rangle d z
$$

and (24) holds true when $\tau \in(0,1)$.

For the cases $\tau=0,1$ we need the operator $J$ defined in (23) and the following equalities which come from easy computations (cf. [24]):

$$
\begin{aligned}
& V_{g} f(x, \omega)=e^{-2 \pi i x \omega} V_{\hat{g}} \hat{f}(\omega,-x), \mathcal{F} T_{x}=M_{-x} \mathcal{F} \\
& \mathcal{F} M_{\omega}=T_{\omega} \mathcal{F}, T_{x} M_{\omega}=e^{-2 \pi i x \omega} M_{\omega} T_{x} .
\end{aligned}
$$

Therefore (24) is proved for $\tau=0,1$ in the following manner. We put $z=(x, \omega)$ and let $\sigma$ acts on functions of variables $(y, \eta)$ :

$$
\begin{aligned}
\left\langle\mathrm{Op}_{0}(\sigma) f, \varphi\right\rangle & =\left\langle\sigma, e^{-2 \pi i y \eta} \varphi(y) \overline{\hat{f}(\eta)}\right\rangle \\
& =\left\langle\sigma, e^{-2 \pi i y \eta} \varphi(y) \int_{\mathbb{R}^{2 d}} \overline{V_{\hat{g}} \hat{f}\left(z^{\prime}\right) \pi\left(z^{\prime}\right) \hat{g}(\eta)} d z^{\prime}\right\rangle \\
& =\left\langle\sigma, \int_{\mathbb{R}^{2 d}} \overline{V_{g} f(z)} e^{-2 \pi i y \eta} \varphi(y) \overline{e^{2 \pi i x \omega} \pi(J z) \hat{g}(\eta)} d z\right\rangle \\
& =\int_{\mathbb{R}^{2 d}} V_{g} f(z)\left\langle\sigma, e^{-2 \pi i y \eta} \varphi(y) \overline{\overline{\pi(z) g}(\eta)}\right\rangle d z \\
& =\int_{\mathbb{R}^{2 d}} V_{g} f(z)\left\langle\sigma, W_{0}(\varphi, \pi(z) g)\right\rangle d z \\
& =\int_{\mathbb{R}^{2 d}} V_{g} f(z)\left\langle\mathrm{Op}_{0}(\sigma) \pi(z) g, \varphi\right\rangle d z .
\end{aligned}
$$

The case $\tau=1$, i.e.

$$
\left\langle\mathrm{Op}_{1}(\sigma) f, \varphi\right\rangle=\int_{\mathbb{R}^{2 d}} V_{g} f(z)\left\langle\mathrm{Op}_{1}(\sigma) \pi(z) g, \varphi\right\rangle d z,
$$

can be proved in the same manner. The details are left to the reader.

\section{References}

1. Bayer, D., Gröchenig, K.: Time-frequency localization operators and a Berezin transform. Integral Equ. Oper. Theory 82(1), 95-117 (2015)

2. Bastianoni, F., Cordero, E., Nicola, F.: Decay and smoothness for eigenfunctions of localization operators. J. Math. Anal. Appl. 492, 124480 (2020)

3. Berezin, F.A.: Wick and anti-Wick symbols of operators. Mat. Sb. (N.S.) 86(128), 578-610 (1971)

4. Berger, C.A., Coburn, L.A.: Toeplitz operators on the Segal-Bargmann space. Trans. Am. Math. Soc. 301(2), 813-829 (1987)

5. Boggiatto, P., Cordero, E., Gröchenig, K.: Generalized anti-Wick operators with symbols in distributional Sobolev spaces. Integral Equ. Oper. Theory 48(4), 427-442 (2004)

6. Cappiello, M., Toft, J.: Pseudo-differential operators in a Gelfand-Shilov setting. Math. Nachr. 290(56), 738-755 (2016) 
7. Chung, J., Chung, S.Y., Kim, D.: Characterizations of the Gelfand-Shilov spaces via Fourier transforms. Proc. Am. Math. Soc. 124, 2101-2108 (1996)

8. Cordero, E.: Gelfand-Shilov window classes for weighted modulation spaces. Integral Trans. Spec. Funct. 18(11), 809-817 (2007)

9. Cordero, E., Gröchenig, K.: Time-frequency analysis of localization operators. J. Funct. Anal. 205(1), 107-131 (2003)

10. Cordero, E., Gröchenig, K.: Necessary conditions for Schatten class localization operators. Proc. Am. Math. Soc. 133(12), 3573-3579 (2005)

11. Cordero, E., Nicola, F., Rodino, L.: A few remarks on time-frequency analysis of Gevrey, analytic and ultra-analytic functions. Four. Anal. 73-90 (2014)

12. Cordero, E., Nicola, F., Trapasso, S.I.: Almost diagonalization of $\tau$-pseudodifferential operators with symbols in Wiener Amalgam and modulation spaces. J. Four. Anal. Appl. 25, 1927-1957 (2019)

13. Cordero, E., Pilipović, S., Rodino, L., Teofanov, N.: Localization operators and exponential weights for modulation spaces. Mediter. J. Math. 2(4), 381-394 (2005)

14. Cordero, E., Pilipović, S., Rodino, L., Teofanov, N.: Quasianalytic Gelfand-Shilov spaces with application to localization operators. Rocky J. Math. 40(4), 1123-1147 (2010)

15. Cordero, E., Rodino, L.: Time-Frequency Analysis of Operators, De Gruyter Studies in Mathematics, vol. 75. Birkhäuser, Boston, Berlin (2020)

16. Daubechies, I.: Time-frequency localization operators: a geometric phase space approach. IEEE Trans. Inf. Theory 34(4), 605-612 (1988)

17. Engliš, M.: Toeplitz operators and localization operators. Trans. Am. Math. Soc. 361(2), 1039-1052 (2009)

18. Feichtinger, H.G.: Modulation spaces on locally compact abelian groups. Wavelets Appl. 99-140 (2003)

19. Folland, G.B.: Harmonic Analysis in Phase Space. Princeton University Press, Princeton (1989)

20. Galperin, Y.V.: Young's convolution inequalities for weighted mixed (quasi-)norm spaces. J. Inequal. Spec. Funct. 5(1), 1-12 (2014)

21. Galperin, Y.V., Samarah, S.: Time-frequency analysis on modulation spaces $M_{m}^{p, q}, 0<p, q \leq \infty$. Appl. Comput. Harmon. Anal. 16(1), 1-18 (2004)

22. Gelfand, I.M., Shilov, G.E.: Generalized Functions. Academic Press, New York, London, pp. I-III (1968)

23. Gramchev, T.: Gelfand-Shilov spaces: structural properties and applications to pseudodifferential operators in $\mathbb{R}^{n}$. In: D. Bahns, W. Bauer, I. Witt (Eds.) Quantization, PDEs, and Geometry, Oper. Theory Adv. Appl. vol. 251, pp. 1-68. Birkhäuser, Basel (2016)

24. Gröchenig, K.: Foundations of Time-Frequency Analysis. Applied and Numerical Harmonic Analysis. Birkhäuser, Boston (2001)

25. Gröchenig, K.: Weight functions in time-frequency analysis. In: Rodino, L., Wong, M.W. (eds.) Pseudodifferential Operators: Partial Differential Equations and Time-Frequency Analysis, vol. 52, pp. 343-366. Fields Institute Communications, American Mathematical Society, Providence (2007)

26. Gröchenig, K., Toft, J.: Isomorphism properties of Toeplitz operators and pseudo-differential operators between modulation spaces. J. Anal. Math. 114, 255-2830 (2011)

27. Gröchenig, K., Toft, J.: The range of localization operators and lifting theorems for modulation and Bargmann-Fock spaces. Trans. Am. Math. Soc. 365(8), 4475-4496 (2013)

28. Gröchenig, K., Zimmermann, G.: Spaces of test functions via the STFT. J. Funct. Spaces Appl. 2(1), 25-53 (2004)

29. Guo, W., Chen, J., Fan, D., Zhao, G.: Characterizations of some properties on weighted modulation and Wiener Amalgam spaces. Mich. Math. J. 68, 451-482 (2019)

30. Kamiński, A., Perišić, D., Pilipović, S.: On various integral transformations of tempered ultradistributions. Demonstr. Math. 33(3), 641-655 (2000)

31. Kobayashi, M., Miyachi, A.: Schatten $p$-class property of pseudodifferential operators with symbols in modulation spaces. Nagoya Math. J. 205, 119-148 (2012)

32. Lozanov-Crvenkovic, Z., Perisic, D., Taskovic, M.: Gelfand-Shilov spaces. Struct. Kernel Theorems. Preprint arXiv:0706.2268v2

33. Molahajloo, S., Okoudjou, K.A., Pfander, G.E.: Boundedness of multilinear pseudodifferential operators on modulation spaces. J. Four. Anal. Appl. 22(6), 1381-1415 (2016)

34. Nicola, F., Rodino, L.: Global Pseudo-Differential Calculus on Euclidean Spaces, Pseudo-Differential Operators, Theory and Applications, vol. 4. Birkhäuser, Basel (2010) 
35. Pilipović, S.: Tempered ultradistributions. Boll. UMI 7-B(2), 235-251 (1988)

36. Ramanathan, J., Topiwala, P.: Time-frequency localization via the Weyl correspondence. SIAM J. Math. Anal. 24(5), 1378-1393 (1993)

37. Shubin, M.A.: Pseudodifferential Operators and Spectral Theory, 2nd edn. Springer, Berlin (2001)

38. Teofanov, N.: Ultradistributions and time-frequency analysis. In: Boggiatto, P., Rodino, L., Toft, J., Wong, M.W. (eds.) Pseudo-Differential Operators and Related Topics, Oper. Theory Adv. Appl., vol. 164, pp. 173-191. Birkhäuser, Basel (2006)

39. Teofanov, N.: Modulation spaces, Gelfand-Shilov spaces and pseudodifferential operators. Sampl. Theory Signal Image Process 5(2), 225-242 (2006)

40. Teofanov, N.: Gelfand-Shilov spaces and localization operators. Funct. Anal. Approx. Comput. 7(2), $135-158(2015)$

41. Teofanov, N.: Continuity and Schatten-von Neumann properties for localization operators on modulation spaces. Mediter. J. Math. 13(2), 745-758 (2016)

42. Toft, J.: Continuity properties for modulation spaces, with applications to pseudo-differential calculus. I. J. Funct. Anal. 207(2), 399-429 (2004)

43. Toft, J.: Continuity properties for modulation spaces, with applications to pseudo-differential calculus. II. Ann. Glob. Anal. Geom. 26(1), 73-106 (2004)

44. Toft, J.: The Bargmann transform on modulation spaces and Gelfand-Shilov spaces, with applications to Toeplitz and pseudo-differential operators. J. Pseudo-Differ. Oper. Appl. 3, 145-227 (2012)

45. Toft, J.: Multiplication properties in Gelfand-Shilov pseudo-differential calculus. Pseudo-differential operators, generalized functions and asymptotics. Oper. Theory: Adv. Appl. 231, 117-172 (2013)

46. Toft, J.: Gabor analysis for a broad class of quasi-Banach modulation spaces. Pseudo-Differ. Oper., General. Funct., Oper. Theory: Adv. Appl. 245, 249-278 (2015)

47. Toft, J.: Continuity and compactness for pseudo-differential operators with symbols in quasi-Banach spaces or Hörmander classes. Anal. Appl. (Singap.) 15(3), 353-389 (2017)

48. Toft, J.: Images of function and distribution spaces under the Bargmann transform. J. Pseudo-Differ. Oper. Appl. 8, 83-139 (2017)

49. Toft, J.: Continuity of Gevrey-Hörmander pseudo-differential operators on modulation spaces. J. Pseudo. Differ. Oper. Appl. 10,337-358 (2019)

50. Wong, M.W.: Wavelet Transforms and Localization Operators, Operator Theory: Advances and Applications, vol. 136. Birkhäuser, Basel (2002)

Publisher's Note Springer Nature remains neutral with regard to jurisdictional claims in published maps and institutional affiliations. 